\title{
1 Common variants contribute to intrinsic human brain functional networks
}

\section{Running title: GWAS of intrinsic brain function}

5 Bingxin Zhao ${ }^{1,14}$, Tengfei $\mathrm{Li}^{2,3,14}$, Stephen M. Smith ${ }^{4}$, Di Xiong ${ }^{1}$, Xifeng Wang ${ }^{1}$, Yue Yang ${ }^{1}$, 6 Tianyou Luo ${ }^{1}$, Ziliang Zhu' ${ }^{1}$, Yue Shan ${ }^{1}$, Nana Matoba ${ }^{5,6}$, Quan Sun ${ }^{1}$, Yuchen Yang ${ }^{5}$, Mads

7 E. Hauberg $7,8,10,11$, Jaroslav Bendl ${ }^{7-9}$, John F. Fullard ${ }^{7-9}$, Panagiotis Roussos ${ }^{7-10,12}$, Weili $8 \operatorname{Lin}^{2,3}$, Yun Li ${ }^{1,5,13}$, Jason L. Stein ${ }^{5,6}$, and Hongtu Zhu ${ }^{1,3 *}$

$10{ }^{1}$ Department of Biostatistics, University of North Carolina at Chapel Hill, Chapel Hill, NC, USA

11 2Department of Radiology, University of North Carolina at Chapel Hill, Chapel Hill, NC, USA

$12{ }^{3}$ Biomedical Research Imaging Center, School of Medicine, University of North Carolina at Chapel Hill,

13 Chapel Hill, NC, USA

$14{ }^{4}$ Wellcome Centre for Integrative Neuroimaging, FMRIB, Nuffield Department of Clinical

15 Neurosciences, University of Oxford, Oxford, UK

$16{ }^{5}$ Department of Genetics, University of North Carolina at Chapel Hill, Chapel Hill, NC, USA

17 UUNC Neuroscience Center, University of North Carolina at Chapel Hill, Chapel Hill, NC, USA

$18{ }^{7}$ Department of Psychiatry, Icahn School of Medicine at Mount Sinai, New York, NY, USA

$19{ }^{8}$ Friedman Brain Institute, Icahn School of Medicine at Mount Sinai, New York, NY, USA

$20{ }^{9}$ Department of Genetics and Genomic Science and Institute for Multiscale Biology, Icahn School of

21 Medicine at Mount Sinai, New York, NY, USA

$22{ }^{10}$ iPSYCH, The Lundbeck Foundation Initiative for Integrative Psychiatric Research, Denmark

$23 \quad{ }^{11}$ Centre for Integrative Sequencing (iSEQ), Aarhus University, Aarhus, Denmark

$24{ }^{12}$ Mental IIIness Research, Education, and Clinical Center (VISN 2 South), James J. Peters VA Medical

25 Center, Bronx, NY, USA

$26{ }^{13}$ Department of Computer Science, University of North Carolina at Chapel Hill, Chapel Hill, NC, USA

$27{ }^{14}$ These authors contributed equally to this work.

$29{ }^{*}$ Corresponding author:

30 Hongtu Zhu

31 3105C McGavran-Greenberg Hall, 135 Dauer Drive, Chapel Hill, NC 27599.

32 E-mail address: htzhu@email.unc.edu Phone: (919) 966-7250 


\section{Abstract}

2 The human brain remains active in the absence of explicit tasks and forms networks of

3 correlated activity. Resting-state functional magnetic resonance imaging (rsfMRI)

4 measures brain activity at rest, which has been linked with both cognitive and clinical

5 outcomes. The genetic variants influencing human brain function are largely unknown.

6 Here we utilized rsfMRI from 44,190 individuals of multiple ancestries (37,339 in the UK

7 Biobank) to discover and validate the common genetic variants influencing intrinsic

8 brain activity. We identified hundreds of novel genetic loci associated with intrinsic

9 functional signatures $\left(P<2.8 \times 10^{-11}\right)$, including associations to the central executive,

10 default mode, and salience networks involved in the triple network model of

11 psychopathology. A number of intrinsic brain activity associated loci colocalized with

12 brain disorder GWAS (e.g., Alzheimer's disease, Parkinson's disease, schizophrenia) and

13 cognition, such as 19q13.32, 17q21.31, and 2p16.1. Particularly, we detected a

14 colocalization between one (rs429358) of the two variants in the APOE \&4 locus and

15 function of the default mode, central executive, attention, and visual networks. Genetic

16 correlation analysis demonstrated shared genetic influences between brain function and

17 brain structure in the same regions. We also detected significant genetic correlations

18 with 26 other complex traits, such as ADHD, major depressive disorder, schizophrenia,

19 intelligence, education, sleep, subjective well-being, and neuroticism. Common variants

20 associated with intrinsic brain activity were enriched within regulatory element in brain

21 tissues.

22

23 Keywords: Amplitude; Functional connectivity; Intrinsic brain activity; GWAS;

24 Resting-state fMRI; Triple network model; UK Biobank. 
1 The human brain is a complex system where functional organization and communication

2 between brain networks are necessary for behavior and cognition ${ }^{1-4}$. The human brain

3 remains active in the absence of explicit tasks or stimuli, resulting in an intrinsic

4 functional architecture. Utilizing changes in blood oxygen level-dependent (BOLD)

5 signal $^{5,6}$, resting-state functional magnetic resonance imaging ${ }^{7}$ (rsfMRI) captures

6 spontaneous intrinsic brain activity ${ }^{8}$. Specifically, the spontaneous neural activity and

7 non-neural physiological processes within each functional region are quantified by the

8 amplitude of low frequency fluctuations (ALFF) in BOLD time series ${ }^{6,9,10}$. Moreover, the

9 inter-regional correlations in spontaneous neuronal variability are used to construct a

10 functional connectivity matrix, which measures the magnitude of temporal synchrony

11 between each pair of brain regions ${ }^{6,11}$.

13 rsfMRI has led to the discovery of multiple resting-state networks (RSNs) present in

14 neurotypical human brains, including the default mode, central executive (i.e., 15 frontoparietal), attention, limbic, salience, somatomotor, and visual networks ${ }^{12-14}$.

16 Among these RSNs, the central executive, default mode, and salience networks are

17 three core neurocognitive networks that support efficient cognition ${ }^{15-17}$. Accumulating

18 evidence suggests that the functional organization and dynamic interaction of these

19 three networks underlie a wide range of mental disorders, resulting in the triple 20 network model of psychopathology ${ }^{16,18}$. Supporting this model, differences in RSNs have

21 been detected in multiple neurological and psychiatric disorders ${ }^{19}$ relative to

22 neurotypical controls, such as Alzheimer's disease ${ }^{20}$, Parkinson's disease ${ }^{21}$, and major

23 depressive disorder (MDD) 22.

25 Twin and family studies have largely reported a low to moderate degree of genetic 26 contributions to intrinsic brain activity ${ }^{23-29}$. For example, the family-based heritability

27 estimates of major RSNs ranged from $20 \%$ to $40 \%$ in the Human Connectome Project $28(\mathrm{HCP})^{30}$. In a previous study using about 8,000 UK Biobank (UKB) individuals ${ }^{31}$, the SNP 29 heritability ${ }^{32}$ of amplitude and functional connectivity traits can be higher than $30 \%$.

30 Although there were multiple candidate gene studies for intrinsic brain activity (such as 31 for $A P O E^{33}$ and $K I B R A^{34}$ ), currently only one genome-wide association study (GWAS) ${ }^{31}$

32 has been successfully performed on $\operatorname{rsfMR}^{23}(n \approx 8000)$. This is likely due to both 
1 insufficient sample size for GWAS discovery and weaker genetic effects on brain

2 function than structure $31,35-39$. It is also known that functional connectivity traits in

3 rsfMRI are typically noisier than brain structural traits measured in other neuroimaging

4 modalities. In addition, imaging batch effects ${ }^{40}$ (e.g., image acquisition, processing

5 procedures, and software) may cause additional technical variability in rsfMRI

6 analyses $^{41}$, making GWAS meta-analysis and independent replication particularly

7 challenging. Therefore, genetic variants influencing intrinsic brain activity have

8 remained largely undiscovered and their shared genetic influences with other complex

9 traits and clinical outcomes are unknown.

11 To address these challenges, here we collected individual-level rsfMRI data from four 12 independent studies, including the UK Biobank ${ }^{42}$, Adolescent Brain Cognitive 13 Development $\left(\mathrm{ABCD}^{43}\right)$, Philadelphia Neurodevelopmental Cohort $\left(\mathrm{PNC}^{44}\right)$, and $\mathrm{HCP}^{45}$.

14 We harmonized rsfMRI processing procedures by following the unified UKB brain 15 imaging pipeline ${ }^{9,46}$. Functional brain regions and corresponding functional connectivity 16 were characterized via spatial Independent Component Analysis (ICA) ${ }^{47,48}$ for 44,190

17 individuals from multiple ancestries, including 37,339 from UK Biobank. As in previous 18 studies $^{9,31,49}$, two parcellations with different dimensionalities ${ }^{13,50}$ (25 and 100 regions, 19 respectively) were separately applied in spatial ICA and we focused on the 76 (21 and 2055 , respectively) regions that had been previously confirmed to be non-artifactual ${ }^{9}$. Two 21 group of neuroimaging phenotypes were then generated: the first group contains 76 22 (node) amplitude traits reflecting the regional spontaneous neuronal activity; and the 23 second group includes 1,695 (i.e., $21 \times 20 / 2+55 \times 54 / 2$ ) (edge) functional connectivity 24 traits that quantify the inter-regional co-activity, as well as 6 global functional 25 connectivity measures summarizing all of the 1,695 pairwise functional connectivity 26 traits $^{31}$. These 1,777 traits were then used to explore the genetic architecture of intrinsic 27 brain activity. To aid interpretation of GWAS results, the functional brain regions 28 characterized in ICA were labelled by using the automated anatomical labeling (AAL) 29 atlas $^{51}$ and were mapped onto major functional networks defined in Yeo, et al. ${ }^{14}$ and 30 Finn, et al. ${ }^{12}$. Our GWAS results can be easily explored and downloaded through the 31 Brain Imaging Genetics Knowledge Portal (BIG-KP) https://bigkp.org. 


\section{RESULTS}

\section{Genetics of the intrinsic brain functional architecture.}

3 SNP heritability was estimated for the 1,777 intrinsic brain activity traits via GCTA ${ }^{52}$. The 4 mean heritability $\left(h^{2}\right)$ estimate was $27.2 \%$ (range $=(10 \%, 36.5 \%)$, standard error $\left.=6.0 \%\right)$

5 for the 76 amplitude traits, all of which remained significant after adjusting for multiple

6 comparisons by using the Benjamini-Hochberg procedure to control false discovery rate

7 (FDR) at 0.05 level (1,777 tests, Fig. 1a and Supplementary Table 1). Among the 1,701

8 functional connectivity traits, 1,230 had significant (again at 5\% FDR) heritability with

9 estimates varying from $3 \%$ to $61 \%$ (mean $=9.6 \%$, standard error $=5.8 \%$ ). Ten functional 10 connectivity traits had heritability higher than 30\%, including 4 global functional

11 connectivity measures (Supplementary Fig. 1) and 6 pairwise functional connectivity

12 traits (Fig. 1b). These most heritable traits were most related to the central executive,

13 default mode, and salience networks in the triple network model of psychopathology ${ }^{16}$.

14 To examine whether intrinsic brain activity within the triple network in general had 15 higher heritability, we classified the 76 amplitude traits into two categories 1) fully or 16 partially within the triple network and 2) outside the triple network. Correspondingly,

17 the 1,695 pairwise functional connectivity traits were classified into 1) within the triple 18 network, 2) outside the triple network, and 3) between the triple and non-triple 19 networks. We found that amplitude traits within the triple network had significantly 20 higher heritability than those outside the triple network (mean $=30.5 \%$ vs. $22.3 \%, P=$ $216.3 \times 10^{-11}$, two-sided Wilcoxon rank test) (Fig. 1c). Similarly, functional connectivity 22 traits within the triple network had higher heritability than interactions outside the 23 triple network or between the triple and non-triple networks (mean $=12.5 \%$ vs. 7\%, $P=$ $\left.241.9 \times 10^{-26}\right)$. These results indicate that the level of genetic control might be higher in 25 core neurocognitive networks. The range of heritability estimates was consistent with 26 previous results ${ }^{31}$, suggesting that common genetic variants had a low to moderate 27 degree of contributions to inter-individual variability of intrinsic brain activity. The 28 overall genetic effects on both amplitude and functional connectivity were lower than 29 those on brain structure. For example, the average heritability was reported to be $48.7 \%$ 30 for diffusion tensor imaging (DTI) traits of brain structural connectivity in white matter 31 tracts $^{53}$ and $40 \%$ for regional brain volumes measuring brain morphometry ${ }^{37}$.

32 Nevertheless, as shown below, intrinsic brain activity may be more functionally relevant 
1 with stronger genetic connections to brain disorders than brain structure, such as

2 Alzheimer's disease.

4 Genome-wide association discovery was carried out for 1,777 intrinsic brain activity 5 traits using UKB individuals of British ancestry ( $n=34,691$, Methods). The Manhattan 6 and QQ plots can be found in the BIG-KP server. At the significance level $2.8 \times 10^{-11}(5 \times$ $710^{-8} / 1,777$, i.e., the standard GWAS threshold, Bonferroni-adjusted for the 1,777 traits), 8 FUMA $^{54}$ identified 264 lead independent variants (linkage disequilibrium [LD] $r^{2}<0.1$ ), 9 and then characterized 606 significant locus-trait associations for 197 traits (75 10 amplitude and 122 functional connectivity (Supplementary Tables 2-3, Supplementary

11 Fig. 2, Methods). The amplitude traits typically had multiple associated variants and a 12 number of variants were widely related to the amplitude in different brain regions, such 13 as rs429358 (nearest gene APOE), rs2274224 (PLCE1), and rs1133400 (INPP5A). In 14 addition, rs2279829 (ZIC4), rs62158211 (AC016745.1), and rs115877304 (NR2F1-AS1) 15 were associated with multiple functional connectivity traits. Global and pairwise 16 functional connectivity traits that had at least 5 significant variants were again most 17 related to the central executive, default mode, and salience networks (Supplementary

18 Fig. 3). Of the 14 associated variants that had been identified in the previous GWAS ${ }^{31}$, 1912 were in $\operatorname{LD}\left(r^{2} \geq 0.6\right)$ with our significant variants, most of which were associated 20 with amplitude traits. In summary, our analyses identify many novel variants associated 21 with intrinsic functional signatures and illustrate the global genetic influences on

22 functional connectivity across the whole brain. The degree of genetic control is higher in 23 the central executive, default mode, and salience networks, whose cross-network 24 interactions closely control multiple cognitive functions and affect major brain 25 disorders ${ }^{18}$.

\section{Replication and the effect of ancestry.}

28 We aimed to replicate our results in UKB British GWAS using other independent 29 datasets. First, we repeated GWAS on UKB individuals of White but Non-British ancestry 30 (UKBW, $n=1,970$ ) and three non-UKB European-ancestry cohorts, including $A B C D$ 31 European (ABCDE, $n=3,821), \operatorname{HCP}(n=495)$, and PNC $(n=510)$. We meta-analyzed the 32 four European GWAS (total $n=6,796$ ) and checked whether the locus-trait associations 
1 detected in UKB British GWAS can be replicated. For the 606 significant associations,

$2101(16.7 \%)$ passed the $8.2 \times 10^{-5}$ (i.e., 0.05/606) Bonferroni significance level in this

3 validation GWAS, and 599 (98.8\%) were significant at FDR 5\% level. Next, we performed

4 GWAS on four non-European validation datasets: the UKB Asian (UKBA, $n=446$ ), UKB

5 Black (UKBBL, $n=232), A B C D$ Hispanic $(A B C D H, n=768)$, and ABCD African American

6 (ABCDA, $n=1,257$ ). We meta-analyzed these four non-European GWAS (total $n=2,703$ )

7 and found that 39 (6.4\%) passed the Bonferroni significance level and 601 (99.2\%) were

8 significant at FDR 5\% level. Some associations with rs3781658 (ANO1), rs7083220

9 (PWWP2B), rs9373978 (FHL5), rs11187838 (PLCE1), and rs35124509 (EPHA3) were

10 replicated in both European and non-European datasets at the stringent Bonferroni

11 significance level. Moreover, we performed a third meta-analysis to combine all of the

12 eight validation datasets, after which the number of replicated associations moved up to

13136 (22.4\%) and 602 (99.3\%) at Bonferroni and FDR significance levels, respectively.

14 These results are summarized in Supplementary Table 4. Overall, our results suggest

15 that the associated genetic loci discovered in UKB British GWAS have high

16 generalizability in independent rsfMRI studies, despite the fact that these studies may

17 use different imaging protocols/MRI scanners and recruit participants from different age

18 groups. The strong homogeneity of GWAS results likely benefit, in part, from the

19 consistent rsfMRI processing procedures that we applied to these datasets.

21 In addition, we utilized polygenic risk scores ${ }^{55}$ (PRS) derived from UKB British GWAS for

22 further evidence of replication (Methods). For the 197 traits that had significant

23 variants, 168 had significant PRS in at least one of the four European validation GWAS

24 datasets at FDR $5 \%$ level $(197 \times 4$ tests, Supplementary Table 5), illustrating the

25 significant out-of-sample prediction power of polygenic influences from our discovery

26 GWAS results. The largest incremental R-squared (after adjusting the effects of age, sex,

27 and ten genetic principal components) were observed on the 2nd, 3rd, 4th, and 6th

28 global functional connectivity measures in UKBW and HCP datasets, which were larger

29 than $5 \%\left(\right.$ range $=(5.1 \%, 5.7 \%), P$ range $\left.=\left(1.1 \times 10^{-24}, 4 \times 10^{-13}\right)\right)$. To evaluate the

30 consistency across ancestry, PRS was also constructed on the four non-European

31 validation datasets. UKBA had the best validation performance among the four datasets,

32 with 86 PRS being significant at FDR 5\% level (197 × 4 tests, Supplementary Table 6). 
1 The number of significant PRS was reduced to 59, 39, and 31 in $A B C D H, A B C D A$, and

2 UKBBL, respectively. In summary, these PRS results illustrate the overall consistency of

3 genetic effects in European cohorts and also show that there may be population specific

4 influences on brain function in other cohorts, though much smaller sample sizes and

5 difficulty in conducting cross ancestry PRS strongly limit the interpretability of these

6 analyses. More efforts are required to identify causal variants associated with functional

7 brain in global diverse populations and perform better cross-population PRS predictions.

9 The shared genetic loci with brain-related complex traits and disorders.

10 To evaluate the shared genetic influences between intrinsic brain activity and other

11 complex traits, we carried out association lookups for independent significant variants

12 (and their LD tags, i.e., variants with LD, $r^{2} \geq 0.6$ ) detected in UKB British GWAS

13 (Methods). In the NHGRI-EBI GWAS catalog ${ }^{56}$, our results tagged many variants reported

14 for a wide range of complex traits in different trait domains, such as neurological and

15 psychiatric disorders, cognitive performance, education, bone mineral density, sleep,

16 smoking/drinking, brain structure, and anthropometric traits. Below we highlighted

17 colocalizations in a few selected genomic regions.

19 The index variants rs429358 (APOE), rs34404554 (TOMM40), rs157582(TOMM40), and

20 rs157592 (APOC1) in the 19q13.32 region (Fig. 2a, Supplementary Fig. 4) had genetic

21 effects on the amplitude of many functional brain regions that were most in the default

22 mode, central executive (i.e., frontoparietal), attention, and visual networks. It is well

23 known that 19q13.32 is a risk locus of Alzheimer's disease and rs429358 is one of the

24 two variants in the $A P O E$ \&4 locus. In this region, we tagged variants associated with

25 dementia and decline in mental ability, including Alzheimer's disease ${ }^{57-59}$,

26 frontotemporal dementia ${ }^{60}$, cerebral amyloid angiopathy ${ }^{61}$, cognitive decline ${ }^{62}$,

27 cognitive impairment test score ${ }^{63}$, as well as many biomarkers of Alzheimer's disease,

28 such as neurofibrillary tangles ${ }^{61}$, neuritic plaque ${ }^{61}$, cerebral amyloid deposition ${ }^{64}$,

29 cerebrospinal fluid protein levels ${ }^{63}$, and cortical amyloid beta load ${ }^{65}$. Altered amplitude

30 activity has been widely reported in patients of cognitive impairment and Alzheimer's

31 disease ${ }^{66,67}$. The brain degeneration related to Alzheimer's disease may begin in the

32 frontoparietal regions $^{68}$ and was associated with dysfunction of multiple RSNs, 
1 especially the default mode network ${ }^{20}$. Our findings suggest the shared genetic

2 influences between intrinsic neuronal activity and brain atrophy of Alzheimer's disease.

$4 \quad$ Next, the variant rs62061845 (KANSL1) in the 17q21.31 region (Supplementary Fig. 5)

5 was associated with functional connectivity over the inferior frontal, middle frontal, 6 superior frontal, middle temporal, and supplementary motor area regions in the default

7 mode and salience networks. Variants in LD with rs62061845 have been frequently

8 reported to be associated with Parkinson's disease studies ${ }^{69-73}$. As a system-level

9 progressive neurodegenerative disorder ${ }^{74}$, Parkinson's disease not only leads to motor

10 abnormalities, but also has non-motor symptoms such as temporal perception

11 abnormalities $^{75}$ and impaired connectivity among frontal regions ${ }^{76}$. Cognitive

12 dysfunction and disrupted coupling between default mode and salience networks were

13 commonly reported in Parkinson's disease ${ }^{17}$. In addition to Parkinson's disease, the

14 17q21.31 region was widely related to other complex traits, including neurological

15 disorders (e.g., Alzheimer's disease ${ }^{77}$, corticobasal degeneration ${ }^{78}$, progressive

16 supranuclear palsy ${ }^{79}$ ), psychiatric disorders (e.g., autism spectrum disorder ${ }^{80}$, depressive

17 symptoms ${ }^{81}$ ), educational attainment ${ }^{82}$, psychological traits (e.g., neuroticism ${ }^{81}$ ),

18 cognitive traits (cognitive ability ${ }^{83}$ ), sleep ${ }^{84}$, heel bone mineral density ${ }^{85}$, alcohol use 19 disorder ${ }^{86}$, subcortical brain volumes ${ }^{38}$, cortical surface area and thickness ${ }^{36}$, and white 20 matter microstructure ${ }^{53}$.

22 In addition, the 2p16.1 (Fig. 2b, Supplementary Fig. 6) and 5q15 (Supplementary Fig. 7)

23 regions were mainly associated with interactions among the central executive, default

24 mode, and salience networks. We observed colocalizations with psychiatric disorders 25 (e.g., schizophrenia ${ }^{87}, \mathrm{MDD}^{88}$, depressive symptoms ${ }^{89}$, autism spectrum disorder ${ }^{90}$ ), 26 psychological traits (e.g., neuroticism ${ }^{81}$, well-being spectrum ${ }^{91}$ ), sleep ${ }^{92}$, cognitive traits

27 (e.g., intelligence ${ }^{93}$ ), and educational attainment ${ }^{82}$. Dysregulated triple network 28 interactions were frequently reported in patients of schizophrenia ${ }^{94}$, depression ${ }^{95}$, and 29 autism spectrum disorder ${ }^{96}$. Similarly, the $2 q 24.2$ (Supplementary Fig. 8) and 10q26.13

30 (Supplementary Fig. 9) regions had genetic effects on functional connectivity traits 31 involved in the central executive, default mode, salience, and limbic networks. In these 32 two regions, our identified variants tagged those that have been implicated with 
1 schizophrenia ${ }^{97}$, educational attainment ${ }^{82}$, cognitive traits (e.g., cognitive ability ${ }^{83}$ ),

2 smoking/drinking (e.g., smoking status ${ }^{98}$, alcohol consumption ${ }^{99}$ ), hippocampus subfield

3 volumes $^{100}$, and heel bone mineral density ${ }^{85}$. We also observed colocalizations in some

4 other genomic regions, such as in 2q14.1 region (Fig. 2c, Supplementary Fig. 10) with

5 sleep traits (e.g., sleep duration ${ }^{84}$, insomnia ${ }^{92}$ ), in 3p11.1 (Supplementary Fig. 11) with

6 cognitive traits (e.g., intelligence ${ }^{101}$, math ability ${ }^{82}$ ), and in 5q14.3 (Supplementary Fig.

7 12) with cognitive traits $^{83}$ and educational attainment ${ }^{82}$. All of these results are

8 summarized in Supplementary Table 7. In summary, intrinsic brain function has wide

9 genetic links to a large number of brain-related complex traits and clinical outcomes,

10 especially neurological and psychiatric disorders and cognitive traits. Integration of

11 GWAS of brain function with these clinical outcomes may help to explain the underlying

12 brain functional mechanisms leading to risk for these disorders.

14 Genetic correlations with brain structure, brain disorders, and cognition.

15 The intricate brain neuroanatomical structure is fundamental in supporting brain

16 function. To explore whether genetically mediated brain structural changes were

17 associated with brain function, we examined pairwise genetic correlations (gc) between

181,777 intrinsic brain activity traits and 315 brain structure traits via LDSC $^{102}$ (Methods),

19 including 100 regional brain volumes ${ }^{37}$ and 215 DTI traits of brain structural connectivity

20 in white matter tracts ${ }^{103}$. There were 151 significant pairs between 94 intrinsic brain

21 functional traits and 73 brain structural traits at FDR $5 \%$ level $(315 \times 1,777$ tests, $|\mathrm{gc}|$

22 range $=(0.22,0.61), P$ range $=\left(1.2 \times 10^{-21}, 1.5 \times 10^{-5}\right)$, Supplementary Table 8$)$.

24 We found significant genetic correlations between regional brain volumes and

25 functional connectivity strengths $\left(|\mathrm{gc}|\right.$ range $=(0.22,0.61), P$ range $=\left(1.2 \times 10^{-21}, 1.2 \times\right.$

$2610^{-5}$ ), Supplementary Fig. 13). Most of the observed correlations were related to higher

27 order brain functional networks, particularly the attention, default mode, salience, and

28 central executive networks. For example, the insula has been widely implicated to be

29 associated with multiple functions, including but not limited to emotion, addiction, and

30 cognition through extensive connections to neocortex, the limbic system, and

31 amygdala ${ }^{104}$. We observed genetic correlations between insula volumes and the

32 connection strengths of multiple pairs of brain regions $(|\mathrm{gc}|$ range $=(0.22,0.27), P<1.2$ 
$1 \times 10^{-5}$, Figs. 3a-b), which were largely in the default mode and central executive 2 networks, including the angular and the inferior and superior frontal regions. Similarly, 3 left inferior parietal lobule volume exhibited strong genetic correlations with 4 connectivity strengths over multiple pairs of brain regions that were known to be a part 5 of the default mode, visual, attention, and salience networks $(|\mathrm{gc}|$ range $=(0.34,0.49)$, $6 \quad P<9.7 \times 10^{-6}$, Supplementary Fig. 14a). Interestingly, however, the above identified 7 genetic correlations appeared to be more specific to the left but not right. The inferior 8 parietal has been implicated to be associated with language function and is connected 9 with the Broca's region via the superior longitudinal fasciculus (SLF) ${ }^{105-107}$. Considering 10 language processing is left-lateralized in about $95 \%$ of right-handers and $75 \%$ of

11 left-handers ${ }^{108-111}$, the observed associations of the left inferior parietal are consistent 12 with the results reported in the literature. In addition, we observed spatial 13 colocalizations between regional brain volumes and their genetically correlated 14 functional connectivity traits in multiple brain regions. For instance, left pericalcarine 15 volume was genetically correlated with the connectivity strengths among its 16 neighboring regions, such as the calcarine, superior occipital, cuneus, precuneus, and 17 lingual, which were largely in the visual, default mode, and central executive networks 18 (Fig. 3c). More spatial overlap/proximity examples included the associations between 19 right precuneus volume and functional connectivity pairs over the precuneus, angular, 20 inferior parietal, and middle temporal regions (Supplementary Fig. 14b); and the

21 associations between postcentral volumes and functional interactions among the 22 postcentral, inferior and superior parietal, supramarginal, and precuneus regions 23 (Supplementary Fig. 14c).

25 Significant genetic correlations were also observed between brain structural 26 connectivity and functional connectivity ||$g c \mid$ range $=(0.25,0.49), P$ range $=\left(5.5 \times 10^{-10}\right.$,

$271.3 \times 10^{-5}$ ), Supplementary Fig. 15). Many of the white matter tracts, in particular the 28 SLF and corpus callosum, manifested a strong genetic correlation with the interactions 29 of functional networks (Fig. 4a). These results provided genetic evidence on how these 30 distributed networks communicate across large distances. The SLF has been widely 31 documented connecting brain regions in temporal, parietal, and frontal lobes ${ }^{112}$. 32 Functionally, SLF has been reported associated with a wide array of brain functions, 
1 including working memory ${ }^{113}$, attention ${ }^{114,115}$, and language functions ${ }^{116,117}$. We

2 observed significant genetic correlations between SLF and connectivity strengths over

3 multiple pairs of brain regions including the frontal, parietal, and temporal regions (|gc|

4 range $=(0.33,0.49), P<2.4 \times 10^{-6}$, Fig. 4b). For example, a significant association

5 between insula and temporal connection and SLF was observed. This finding is

6 consistent with the well documented broad functions of insula, including attention and

7 salience processes ${ }^{104}$. Furthermore, parietal and frontal connections most likely

8 reflected attention and executive control networks. Moreover, the splenium of corpus

9 callosum (SCC) is located in the most posterior part of the corpus callosum and connects

10 brain regions in the temporal, posterior parietal, and occipital lobes. Our results show

11 that SCC was genetically associated with brain regions within the parietal lobe (|gc|

12 range $=(0.34,0.48), P<6.5 \times 10^{-6}$, Fig. $\left.4 \mathrm{c}\right)$. In particular, multiple regions connected to

13 the precuneus were observed, such as the inferior parietal, supramarginal, and occipital

14 regions. The precuneus has been shown to connect multiple cortical and subcortical

15 regions. Functionally, the precuneus is one of the critical areas of the default mode

16 network and has also been implicated to be associated with attention as well as

17 memory functions ${ }^{118}$. Our findings suggest that these connections may be genetically

18 mediated by the SCC. Besides functional connectivity traits, amplitude traits also had

19 significant genetic associations with regional brain volumes and white matter tracts

20 (Supplementary Figs. 16-17, Supplementary Note). Overall, our results uncover the

21 genetic links between intrinsic brain function networks and the associated structural

22 substrates. As illustrated, a few pairs of the genetically correlated brain functional and

23 structural traits show high congruity in spatial location and the involved functions. There

24 has been growing interest to understand how brain topography interacts with brain

25 functional networks ${ }^{119}$. To our knowledge, our results are the first to indicate that

26 genetic changes in brain structure may also impact brain function.

28 Next, we examined the genetic correlations between 1,777 intrinsic brain activity traits 29 and 30 other complex traits, mainly focusing on brain disorders and cognition

30 (Supplementary Table 9). We found 176 significant pairs between 26 complex traits and

31102 intrinsic brain activity traits at FDR $5 \%$ level $\left(30 \times 1,777\right.$ tests, $P$ range $=\left(8.6 \times 10^{-12}\right.$,

$322.3 \times 10^{-3}$ ), Supplementary Table 10). Particularly, functional connectivity strengths 
1 were genetically correlated with a few brain disorders, including attention deficit 2 hyperactivity disorder (ADHD), schizophrenia (SCZ), major depressive disorder (MDD),

3 and cross disorder (five major psychiatric disorders $\left.{ }^{120}\right)(|\mathrm{gc}|$ range $=(0.18,0.37), P<1.2$

$4 \times 10^{-4}$, Fig. 5a). For example, we observed a significant genetic correlation between

5 ADHD and functional interactions among the precentral, supplementary motor area,

6 superior frontal, putamen, and caudate regions, which were largely in the attention,

7 salience, motor, and subcortical-cerebellum networks (Fig. 5b). These brain regions

8 have been widely implicated with ADHD in previous studies. ADHD patients have been

9 observed to have stronger connectivity across the supplementary motor area,

10 precentral, and superior frontal regions ${ }^{121}$. These regions are also associated with

11 difficulties in performing some fine motor skills ${ }^{122}$. In addition, the putamen and

12 caudate regions compose the dorsal striatum, one largest part of the basal ganglia,

13 which is important in controlling motor functions ${ }^{123,124}$. Moreover, significant genetic

14 correlations were observed between SCZ and connection strengths over the precentral,

15 postcentral, precuneus, frontal, and superior parietal regions (Fig. 5c); and between

16 MDD and the interactions among the middle temporal, angular, and superior and

17 middle frontal regions (Fig. 5d, Supplementary Note).

19 In addition, many genetic correlations were observed between functional connectivity 20 and cognitive traits studied in previous GWAS, including intelligence, cognitive 21 performance, general cognitive function, and numerical reasoning. For example,

22 intelligence had genetic correlations with connection strengths over multiple brain

23 regions $\left(|\mathrm{gc}|\right.$ range $=(0.11,0.34), P<1.8 \times 10^{-4}$, Fig. 5e). The strongest correlation

24 located at the superior and middle frontal regions in the central executive and salience

25 networks. It is known that the frontal lobe is associated with higher level cognitive skills,

26 such as problem solving, thinking, planning, and organizing ${ }^{125}$. Wang, et al. ${ }^{126}$ revealed a

27 general intelligence network for logical-math, general intelligence, and linguistic skills,

28 which widely included frontal, parietal, occipital, temporal, and limbic regions.

29 Furthermore, significant genetic correlations were broadly observed on subjective

30 well-being, education, neuroticism, sleep, risk tolerance, automobile speeding, manual

31 occupation, BMI, high blood pressure, and behavioral factors (drinking and smoking) 
1 (Supplementary Figs. 18-19). More details and interpretations can be found in

2 Supplementary Note.

4 Gene-level association analysis and biological annotations.

5 Gene-level association was tested via MAGMA ${ }^{127}$ (Methods), which detected 970

6 significant gene-trait associations $\left(P<1.5 \times 10^{-9}\right.$, adjusted for 1,777 phenotypes $)$ for 123

7 genes (Supplementary Fig. 20, Supplementary Table 11). In addition, we applied

8 FUMA $^{54}$ to map significant variants $\left(P<2.8 \times 10^{-11}\right)$ to genes via physical position,

9 expression quantitative trait loci (eQTL) association, and 3D chromatin (Hi-C)

10 interaction, which yielded 197 more associated genes that were not discovered in

11 MAGMA (276 in total, Supplementary Table 12). For the 320 genes associated with

12 intrinsic brain activity in either MAGMA or FUMA, 84 had been linked to white matter

13 microstructure ${ }^{103}, 48$ were reported to be associated with regional brain volumes ${ }^{37}$, and

1442 were related to both of them (Supplementary Table 13). These triple overlapped

15 genes were also widely associated with other complex traits, such as Parkinson's disease,

16 neuroticism, stroke, alopecia, handedness, and intelligence (Supplementary Table 14),

17 providing more insights into the genetic overlaps among brain structure, brain function,

18 and other brain-related traits. For example, MAPT, NSF, WNT3, and LRRC37A3 were risk

19 genes of Parkinson's disease, which were also associated with pallidum volumes ${ }^{37}$, white

20 matter microstructure ${ }^{103}$, and intrinsic functional connectivity in central executive,

21 default mode, and salience networks. These complementary neuroimaging traits had all

22 been used to study the pathophysiology of Parkinson's disease ${ }^{128-130}$. Similarly, CDKN2C

23 and FAF1 were associated with ischemic stroke ${ }^{131}$ as well as multiple neuroimaging

24 traits of brain structure and function. In addition, 4 of our intrinsic brain activity

25 associated genes (CALY, SLC47A1, CYP2C8, and CYP2C9) were targets for 11 nervous

26 system drugs ${ }^{132}$, such as 4 psycholeptics (ATC code: N05) to produce calming effects, 2

27 anti-depressants (N06A) to treat MDD and related conditions, 2 anti-migraine (N02C),

28 and one anti-dementia (N06D) (Supplementary Table 15).

30 It is of particular interest to study the functional connectivity dysfunction in Alzheimer's

31 disease and identify the overlapped genes ${ }^{20,133}$. Our gene-level analysis replicated APOE

32 and SORL1, which were frequently targeted in Alzheimer's disease-candidate gene 
1 studies of functional connectivity ${ }^{23,134}$. More importantly, we uncovered more

2 overlapped genes between intrinsic brain activity and Alzheimer's disease, such as

3 PVRL2, TOMM40, APOC1, MAPK7, CLPTM1, HESX1, BCAR3, ANO3, and YAP1

4 (Supplementary Table 16). Interestingly, through the BIG-KP server, we found that

5 these genes had much stronger associations with intrinsic brain function than brain

6 structure. We also observed many pleiotropic genes associated with serum metabolite,

7 low density lipoprotein cholesterol, high density lipoprotein cholesterol, triglyceride,

8 type II diabetes mellitus, and blood protein measurements, all of which might be related

9 to the Alzheimer's disease ${ }^{135,136}$. These results expand the overview of the shared

10 genetic components among metabolic dysfunction, blood biomarkers, brain function in

11 Alzheimer's disease research, suggesting the potential value of integrating these traits in

12 future studies.

14 To identify the tissues and cell types in which genetic variation yields differences in brain

15 functional connectivity, we performed partitioned heritability analyses ${ }^{137}$ for tissue type 16 and cell type specific regulatory elements ${ }^{138}$ (Methods). We focused on the 10

17 functional connectivity traits that had heritability higher than $30 \%$. At FDR $5 \%$ level, the

18 most significant enrichments of heritability were observed in active gene regulation

19 regions of fetal brain tissues, neurospheres, and neuron/neuronal progenitor cultured

20 cells (Supplementary Fig. 21, Supplementary Table 17). We also tried to further identify

21 brain cell type specific enrichments using chromatin accessibility data of two main gross

22 brain cell types ${ }^{139}$ (i.e., neurons $(\mathrm{NeuN}+$ ) and glia (NeuN-)) and multiple neuronal and

23 glial cell subtypes, including oligodendrocyte (NeuN-/Sox10+), microglia, and astrocyte

24 (NeuN-/Sox10-), as well as GABAergic (NeuN+/Sox6+) and glutamatergic neurons

25 (NeuN+/Sox6-). Although enrichments were observed in some cell types, few of them

26 remained significant after adjusting for multiple testing (Supplementary Fig. 22,

27 Supplementary Table 18). Next, we performed MAGMA tissue-specific gene property ${ }^{127}$

28 analysis for 13 GTEx $^{140}$ (v8) brain tissues (Methods). We found that genes with higher

29 expression levels in human brain tissues generally had stronger associations with

30 intrinsic brain activity, particularly for tissues sampled from cerebellar hemisphere and

31 cerebellum regions ( $P<1.9 \times 10^{-5}$, Supplementary Fig. 23, Supplementary Table 19). 
1 Among the associated variants of intrinsic brain activity, a few resided in frequently

2 interacting regions (FIREs) and topologically associating domain (TAD) boundaries in

3 brain tissues ${ }^{141,142}$ (Supplementary Table 20). Partitioned heritability analysis also

4 provided suggestive evidence of heritability enrichment in these FIREs and TAD

5 boundaries (Supplementary Fig. 24, Supplementary Table 21). We performed

6 additional gene mapping using 14 recent $\mathrm{Hi}-\mathrm{C}$ datasets of brain tissue and cell

7 types $^{141-145}$ (Methods). This Hi-C gene mapping prioritized 29 genes, 14 of which were

8 not identified by the $\mathrm{Hi}-\mathrm{C}$ analysis in $\mathrm{FUMA}^{54}$ (Supplementary Table 22). Many of the

9 newly mapped genes have been reported for brain-related disorders/conditions, sleep,

10 and intelligence, including APOE, HSPG2, APOC1, UFL1, NR2F1, NPM1, FAM172A, FADD,

11 FHL5, and EPHA3. Finally, MAGMA ${ }^{127}$ gene-set analysis was performed to prioritize the

12 enriched biological pathways (Methods). We found 59 significantly enriched gene sets

13 after Bonferroni adjustment $\left(P<1.8 \times 10^{-9}\right.$, Supplementary Table 23). Multiple

14 pathways related to nervous system were detected, such as "go neurogenesis" (GO:

15 0022008), "go neuron differentiation" (GO: 0030182), "go regulation of nervous system

16 development" (GO: 0051960), "go regulation of neuron differentiation” (GO: 0045664),

17 "go cell morphogenesis involved in neuron differentiation" (GO: 0048667), and "go

18 neuron development" (GO: 0048666).

\section{DISCUSSION}

21 In the present study, we evaluated the influences of common variants on intrinsic brain

22 functional architecture using harmonized rsfMRI data of 44,190 subjects from four

23 independent studies. Genome-wide association analysis found hundreds of novel loci

24 related to intrinsic brain activity in the UKB British cohort, which were successfully

25 replicated in independent datasets. The interactions across core neurocognitive

26 networks (central executive, default mode, and salience) in the triple network model

27 had genetic links with cognition and multiple brain disorders. Shared genetic influences

28 among functional, structural, and diffusion neuroimaging traits were also uncovered,

29 showing that brain structure and function are intimately related. Gene-level analysis

30 detected many overlapped genes between intrinsic brain activity and Alzheimer's

31 disease. We also detected a colocalization between one of the two variants in the APOE

$32 \varepsilon 4$ locus and function of the default mode, central executive, attention, and visual 
1 networks, which may explain in part the functional mechanism underlying Alzheimer's

2 risk. The enriched tissues and biological pathways were also prioritized in bioinformatic

3 analyses. Compared to the previous study ${ }^{31}$ with about 8,000 subjects, this large-scale

4 GWAS much improved our understanding of the genetic architecture of functional

5 human brain.

7 Our study faces a few limitations. First, the samples in our discovery GWAS were mainly

8 from European ancestry. In our PRS analysis, we illustrated a relatively poor replication

9 of the European GWAS results within validation cohorts with non-European ancestry.

10 The non-European GWAS was of small sample size, so population specific influences will

11 be better understood when more data from global populations become available.

12 Second, our study focused on the brain functional activity at rest. A recent study ${ }^{28}$ had

13 found that combining rsfMRI and task functional magnetic resonance imaging (tfMRI)

14 may result in higher heritability estimates and potentially boost the GWAS power. Thus,

15 future studies could model rsfMRI and tfMRI together to uncover more insights into the

16 genetic influences on brain function. In addition, we applied ICA in this study, which was

17 a popular approach to characterize the functionally connected brain ${ }^{6}$. It is also of great

18 interest to evaluate the performance of other popular rsfMRI approaches (such as

19 seed-based analysis) in these large-scale datasets. Finally, although we found genetic

20 links between brain function and other complex traits, future work is needed to dissect

21 the underlying mechanisms by which genetic variation leads to differences in brain

22 activity. We expect that accumulating publicly available imaging genetics data resources

23 will lead to a better understanding of specific genes involved in human brain structure

24 function relationships and how variants can alter these relationships leading to risk for

25 neuropsychiatric disorders.

\section{URLS.}

28 Brain Imaging Genetics Knowledge Portal (BIG-KP), https://bigkp.org/;

29 Brain Imaging GWAS Summary Statistics, https://github.com/BIG-S2/GWAS;

30 UKB Imaging Pipeline, https://git.fmrib.ox.ac.uk/falmagro/UK biobank pipeline v 1;

31 PLINK, https://www.cog-genomics.org/plink2/;

32 GCTA \& fastGWA, http://cnsgenomics.com/software/gcta/; 
1 METAL, https://genome.sph.umich.edu/wiki/METAL;

2 FUMA, http://fuma.ctglab.nl/;

3 MGAMA, https://ctg.cncr.nl/software/magma;

4 LDSC, https://github.com/bulik/ldsc/;

5 FINDOR, https://github.com/gkichaev/FINDOR;

6 NHGRI-EBI GWAS Catalog, https://www.ebi.ac.uk/gwas/home;

7 The atlas of GWAS Summary Statistics, http://atlas.ctglab.nl/.

9 METHODS

10 Methods are available in the Methods section.

11 Note: One supplementary information pdf file and one supplementary table zip file are

12 available.

\section{ACKNOWLEDGEMENTS}

15 This research was partially supported by U.S. NIH grants MH086633 (H.Z.) and

$16 \mathrm{MH} 116527$ (TF.L.). We thank the individuals represented in the UK Biobank, ABCD, HCP,

17 and PNC studies for their participation and the research teams for their work in 18 collecting, processing and disseminating these datasets for analysis. We gratefully 19 acknowledge all the studies and databases that made GWAS summary data available. 20 This research has been conducted using the UK Biobank resource (application number 21 22783), subject to a data transfer agreement. Part of the data used in the preparation of 22 this article were obtained from the Adolescent Brain Cognitive Development (ABCD) 23 Study (https://abcdstudy.org), held in the NIMH Data Archive (NDA). This is a multisite,

24 longitudinal study designed to recruit more than 10,000 children age 9-10 and follow 25 them over 10 years into early adulthood. The ABCD Study is supported by the National 26 Institutes of Health and additional federal partners under award numbers 27 U01DA041022, U01DA041028, U01DA041048, U01DA041089, U01DA041106, 28 U01DA041117, U01DA041120, U01DA041134, U01DA041148, U01DA041156, 29 U01DA041174, U24DA041123, U24DA041147, U01DA041093, and U01DA041025. A full 30 list of supporters is available at https://abcdstudy.org/federal-partners.html. A listing of 31 participating sites and a complete listing of the study investigators can be found at 32 https://abcdstudy.org/scientists/workgroups/. ABCD consortium investigators designed 
1 and implemented the study and/or provided data but did not necessarily participate in

2 analysis or writing of this report. This manuscript reflects the views of the authors and

3 may not reflect the opinions or views of the NIH or $A B C D$ consortium investigators.

4 Support for the collection of the PNC datasets was provided by grant RC2MH089983

5 awarded to Raquel Gur and RC2MH089924 awarded to Hakon Hakonarson. All PNC

6 subjects were recruited through the Center for Applied Genomics at The Children's

7 Hospital in Philadelphia. HCP data were provided by the Human Connectome Project,

8 WU-Minn Consortium (Principal Investigators: David Van Essen and Kamil Ugurbil;

9 1U54MH091657) funded by the 16 NIH Institutes and Centers that support the NIH

10 Blueprint for Neuroscience Research; and by the McDonnell Center for Systems

11 Neuroscience at Washington University.

\section{AUTHOR CONTRIBUTIONS}

14 B.Z., H.Z., J.L.S., S.M.S., and Y.L. designed the study. B.Z., TF.L., D.X., X.W., Y.Y., TY.L., 15 N.M., Q.S., YC.Y. analyzed the data. TF. L., Z.Z., and Y.S. downloaded the datasets, 16 processed rsfMRI data, and undertook quantity controls. P.R., M.E.H., J.B., and J.F.F. 17 analyzed brain cell chromatin accessibility data. B.Z. and H.Z. wrote the manuscript with 18 feedback from all authors.

CORRESPINDENCE AND REQUESTS FOR MATERIALS should be addressed to H.Z.

\section{COMPETETING FINANCIAL INTERESTS}

23 The authors declare no competing financial interests.

\section{REFERENCES}

27 1. Petersen, S.E. \& Sporns, O. Brain networks and cognitive architectures. Neuron

28 88, 207-219 (2015).

$292 . \quad$ Park, H.-J. \& Friston, K. Structural and functional brain networks: from connections to cognition. Science 342(2013).

31 3. Medaglia, J.D., Lynall, M.-E. \& Bassett, D.S. Cognitive network neuroscience. Journal of cognitive neuroscience 27, 1471-1491 (2015). 
14 van den Heuvel, M.P. \& Sporns, O. Network hubs in the human brain. Trends in

$2 \quad$ cognitive sciences 17, 683-696 (2013).

35 Fox, M.D. \& Greicius, M. Clinical applications of resting state functional

4 connectivity. Frontiers in systems neuroscience 4, 19 (2010).

56 LV, H. et al. Resting-state functional MRI: everything that nonexperts have always

$6 \quad$ wanted to know. American Journal of Neuroradiology 39, 1390-1399 (2018).

7 7. Biswal, B., Zerrin Yetkin, F., Haughton, V.M. \& Hyde, J.S. Functional connectivity

8 in the motor cortex of resting human brain using echo-planar MRI. Magnetic

9 resonance in medicine 34, 537-541 (1995).

10 8. Fox, M.D. \& Raichle, M.E. Spontaneous fluctuations in brain activity observed

11 with functional magnetic resonance imaging. Nature reviews neuroscience 8,

12 700-711 (2007).

13 9. Alfaro-Almagro, F. et al. Image processing and Quality Control for the first 10,000

14 brain imaging datasets from UK Biobank. Neurolmage 166, 400-424 (2018).

15 10. Jia, X.-Z. et al. Percent amplitude of fluctuation: a simple measure for resting-state fMRI signal at single voxel level. Plos one 15, e0227021 (2020).

17 11. Hutchison, R.M. et al. Dynamic functional connectivity: promise, issues, and interpretations. Neuroimage 80, 360-378 (2013).

12. Finn, E.S. et al. Functional connectome fingerprinting: identifying individuals using patterns of brain connectivity. Nature neuroscience 18, 1664-1671 (2015).

21 13. Smith, S.M. et al. Correspondence of the brain's functional architecture during activation and rest. Proceedings of the national academy of sciences 106, 13040-13045 (2009).

14. Yeo, B.T. et al. The organization of the human cerebral cortex estimated by intrinsic functional connectivity. Journal of neurophysiology (2011).

15. Menon, V. \& Uddin, L.Q. Saliency, switching, attention and control: a network model of insula function. Brain Structure and Function 214, 655-667 (2010).

28 16. Menon, V. Large-scale brain networks and psychopathology: a unifying triple network model. Trends in cognitive sciences 15, 483-506 (2011). 
disease. Journal of the International Neuropsychological Society: JINS 22, 205 (2016).

18. Menon, V. The triple network model, insight, and large-scale brain organization in autism. Biological psychiatry 84, 236 (2018).

19. Lee, M.H., Smyser, C.D. \& Shimony, J.S. Resting-state fMRI: a review of methods and clinical applications. American Journal of neuroradiology 34, 1866-1872 (2013).

20. Badhwar, A. et al. Resting-state network dysfunction in Alzheimer's disease: a systematic review and meta-analysis. Alzheimer's \& Dementia: Diagnosis, Assessment \& Disease Monitoring 8, 73-85 (2017).

21. Wolters, A.F. et al. Resting-state $\mathrm{fMRI}$ in Parkinson's disease patients with cognitive impairment: A meta-analysis. Parkinsonism \& Related Disorders 62, 16-27 (2019).

22. Mulders, P.C., van Eijndhoven, P.F., Schene, A.H., Beckmann, C.F. \& Tendolkar, I. Resting-state functional connectivity in major depressive disorder: a review. Neuroscience \& Biobehavioral Reviews 56, 330-344 (2015).

23. Foo, H. et al. Genetic influence on ageing-related changes in resting-state brain functional networks in healthy adults: a systematic review. Neuroscience \& Biobehavioral Reviews (2020).

24. Teeuw, J. et al. Genetic and environmental influences on functional connectivity within and between canonical cortical resting-state networks throughout adolescent development in boys and girls. Neurolmage 202, 116073 (2019).

25. Meda, S.A. et al. Multivariate analysis reveals genetic associations of the resting default mode network in psychotic bipolar disorder and schizophrenia. Proceedings of the National Academy of Sciences 111, E2066-E2075 (2014).

26. Duncan, L. et al. Analysis of polygenic risk score usage and performance in diverse human populations. Nature communications 10, 1-9 (2019).

27. Glahn, D. et al. Genetic control over the resting brain. Proceedings of the National Academy of Sciences 107, 1223-1228 (2010).

28. Elliott, M.L. et al. General functional connectivity: Shared features of resting-state and task $\mathrm{fMRI}$ drive reliable and heritable individual differences in functional brain networks. Neurolmage 189, 516-532 (2019). 
1 29. Ge, T., Holmes, A.J., Buckner, R.L., Smoller, J.W. \& Sabuncu, M.R. Heritability analysis with repeat measurements and its application to resting-state functional connectivity. Proceedings of the National Academy of Sciences 114, 5521-5526

4 (2017).

5 30. Adhikari, B.M. et al. Heritability estimates on resting state fMRI data using ENIGMA analysis pipeline. (2018).

31. Elliott, L.T. et al. Genome-wide association studies of brain imaging phenotypes

9 32. Yang, J., Zeng, J., Goddard, M.E., Wray, N.R. \& Visscher, P.M. Concepts, estimation and interpretation of SNP-based heritability. Nature Genetics 49,

12 33. Chiesa, P.A. et al. Revolution of resting-state functional neuroimaging genetics in Alzheimer's disease. Trends in neurosciences 40, 469-480 (2017).

34. Zhang, N. et al. APOE and KIBRA interactions on brain functional connectivity in healthy young adults. Cerebral Cortex 27, 4797-4805 (2017).

16 35. Satizabal, C.L. et al. Genetic architecture of subcortical brain structures in 38,851 individuals. Nature genetics 51, 1624-1636 (2019).

36. Grasby, K.L. et al. The genetic architecture of the human cerebral cortex. Science 367(2020).

37. Zhao, B. et al. Genome-wide association analysis of 19,629 individuals identifies variants influencing regional brain volumes and refines their genetic co-architecture with cognitive and mental health traits. Nature Genetics 51, 1637-1644 (2019).

38. Hibar, D.P. et al. Common genetic variants influence human subcortical brain structures. Nature 520, 224-229 (2015).

39. Stein, J.L. et al. Identification of common variants associated with human hippocampal and intracranial volumes. Nature Genetics 44, 552-561 (2012).

28 40. Smith, S.M. \& Nichols, T.E. Statistical challenges in "big data" human neuroimaging. Neuron 97, 263-268 (2018). dataset by many teams. Nature, 1-7 (2020). 
42. Sudlow, C. et al. UK biobank: an open access resource for identifying the causes of a wide range of complex diseases of middle and old age. PLoS Medicine 12, e1001779 (2015).

43. Casey, B. et al. The adolescent brain cognitive development ( $A B C D)$ study: imaging acquisition across 21 sites. Developmental cognitive neuroscience 32, 43-54 (2018).

44. Satterthwaite, T.D. et al. Neuroimaging of the Philadelphia neurodevelopmental cohort. Neuroimage 86, 544-553 (2014).

45. Somerville, L.H. et al. The Lifespan Human Connectome Project in Development: A large-scale study of brain connectivity development in 5-21 year olds. Neurolmage 183, 456-468 (2018).

46. Miller, K.L. et al. Multimodal population brain imaging in the UK Biobank prospective epidemiological study. Nature Neuroscience 19, 1523-1536 (2016).

47. Beckmann, C.F. \& Smith, S.M. Probabilistic independent component analysis for functional magnetic resonance imaging. IEEE transactions on medical imaging 23, 137-152 (2004).

48. Hyvarinen, A. Fast and robust fixed-point algorithms for independent component analysis. IEEE transactions on Neural Networks 10, 626-634 (1999).

49. Shen, X. et al. Resting-state connectivity and its association with cognitive performance, educational attainment, and household income in the UK Biobank. Biological Psychiatry: Cognitive Neuroscience and Neuroimaging 3, 878-886 (2018).

50. Smith, S.M. et al. Resting-state $\mathrm{fMRI}$ in the human connectome project. Neuroimage 80, 144-168 (2013).

51. Rolls, E.T., Huang, C.-C., Lin, C.-P., Feng, J. \& Joliot, M. Automated anatomical labelling atlas 3. Neurolmage 206, 116189 (2020).

52. Yang, J., Lee, S.H., Goddard, M.E. \& Visscher, P.M. GCTA: a tool for genome-wide complex trait analysis. The American Journal of Human Genetics $\mathbf{8 8}, \mathbf{7 6 - 8 2}$ (2011).

53. Zhao, B. et al. Large-scale GWAS reveals genetic architecture of brain white matter microstructure and genetic overlap with cognitive and mental health traits $(n=17,706)$. Molecular Psychiatry (2019). 
1 54. Watanabe, K., Taskesen, E., Bochoven, A. \& Posthuma, D. Functional mapping and annotation of genetic associations with FUMA. Nature Communications $\mathbf{8}$, 1826 (2017).

4 55. Jansen, I.E. et al. Genome-wide meta-analysis identifies new loci and functional pathways influencing Alzheimer's disease risk. Nature genetics 51, 404-413 (2019).

56. Buniello, A. et al. The NHGRI-EBI GWAS Catalog of published genome-wide association studies, targeted arrays and summary statistics 2019. Nucleic Acids Research 47, D1005-D1012 (2018).

57. Jun, G.R. et al. Transethnic genome-wide scan identifies novel Alzheimer's disease loci. Alzheimer's \& Dementia 13, 727-738 (2017).

58. Nazarian, A., Yashin, A.I. \& Kulminski, A.M. Genome-wide analysis of genetic predisposition to Alzheimer's disease and related sex disparities. Alzheimer's

59. research \& therapy 11, 5 (2019). PICALM associated with Alzheimer's disease. Nature genetics 41, 1088 (2009).

60. Ferrari, R. et al. A genome-wide screening and SNPs-to-genes approach to identify novel genetic risk factors associated with frontotemporal dementia. Neurobiology of aging 36, 2904. e13-2904. e26 (2015).

61. Beecham, G.W. et al. Genome-wide association meta-analysis of neuropathologic features of Alzheimer's disease and related dementias. PLoS Genet 10, e1004606 (2014).

62. Davies, G. et al. A genome-wide association study implicates the APOE locus in nonpathological cognitive ageing. Molecular Psychiatry 19, 76-87 (2014).

63. Liu, C. et al. Genome-wide association and mechanistic studies indicate that

64. Yan, Q. et al. Genome-wide association study of brain amyloid deposition as measured by Pittsburgh Compound-B (PiB)-PET imaging. Molecular psychiatry,

31 65. Scelsi, M.A. et al. Genetic study of multimodal imaging Alzheimer's disease progression score implicates novel loci. Brain 141, 2167-2180 (2018). 
$166 . \quad$ Liang, P. et al. Altered amplitude of low-frequency fluctuations in early and late mild cognitive impairment and Alzheimer's disease. Current Alzheimer Research

$3 \quad 11,389-398(2014)$.

4 67. Liu, X. et al. Abnormal amplitude of low-frequency fluctuations of intrinsic brain

5 activity in Alzheimer's disease. Journal of Alzheimer's Disease 40, 387-397 (2014).

6 68. Mattsson, N. et al. Emerging $\beta$-amyloid pathology and accelerated cortical atrophy. JAMA neurology 71, 725-734 (2014).

8 69. Edwards, T.L. et al. Genome-wide association study confirms SNPs in SNCA and 9 the MAPT region as common risk factors for Parkinson disease. Annals of Human $10 \quad$ Genetics 74, 97-109 (2010).

11 70. Pickrell, J.K. et al. Detection and interpretation of shared genetic influences on 42 human traits. Nature genetics 48, 709 (2016).

13 71. Consortium, I.P.D.G. Imputation of sequence variants for identification of genetic risks for Parkinson's disease: a meta-analysis of genome-wide association

72. Vacic, V. et al. Genome-wide mapping of IBD segments in an Ashkenazi PD cohort identifies associated haplotypes. Human molecular genetics 23, 4693-4702 (2014).

73. Pankratz, N. et al. Meta-analysis of Parkinson's disease: identification of a novel

74. Caligiore, D. et al. Parkinson's disease as a system-level disorder. npj Parkinson's Disease 2, 1-9 (2016).

23 75. Bernardinis, M., Atashzar, S.F., Jog, M.S. \& Patel, R.V. Differential Temporal Perception Abilities in Parkinson's Disease Patients Based on Timing Magnitude.

76. Rowe, J. et al. Attention to action in Parkinson's disease: impaired effective connectivity among frontal cortical regions. Brain 125, 276-289 (2002).

77. Jun, G. et al. A novel Alzheimer disease locus located near the gene encoding tau protein. Molecular Psychiatry 21, 108-117 (2016).

78. Kouri, N. et al. Genome-wide association study of corticobasal degeneration identifies risk variants shared with progressive supranuclear palsy. Nature communications 6, 7247 (2015). 
1 79. Höglinger, G.U. et al. Identification of common variants influencing risk of the tauopathy progressive supranuclear palsy. Nature genetics 43, 699 (2011).

3 80. Grove, J. et al. Identification of common genetic risk variants for autism 4 spectrum disorder. Nature genetics 51, 431 (2019).

5 81. Nagel, M. et al. Meta-analysis of genome-wide association studies for neuroticism in 449,484 individuals identifies novel genetic loci and pathways. Nature Genetics 50, 920 (2018).

8 82. Lee, J.J. et al. Gene discovery and polygenic prediction from a genome-wide association study of educational attainment in 1.1 million individuals. Nature Genetics 50, 1112-1121 (2018).

11 83. Davies, G. et al. Genome-wide association study of cognitive functions and educational attainment in UK Biobank ( $N=112$ 151). Molecular Psychiatry 21, 758-767 (2016).

84. Dashti, H. et al. GWAS in 446,118 European adults identifies 78 genetic loci for self-reported habitual sleep duration supported by accelerometer-derived estimates. bioRxiv, 274977 (2018).

85. Morris, J.A. et al. An atlas of genetic influences on osteoporosis in humans and mice. Nature genetics 51, 258 (2019).

86. Sanchez-Roige, S. et al. Genome-wide association study meta-analysis of the Alcohol Use Disorders Identification Test (AUDIT) in two population-based cohorts. American Journal of Psychiatry, appi. ajp. 2018.18040369 (2018).

87. Pardiñas, A.F. et al. Common schizophrenia alleles are enriched in mutation-intolerant genes and in regions under strong background selection. Nature Genetics 50, 381-389 (2018).

88. Hyde, C.L. et al. Identification of 15 genetic loci associated with risk of major depression in individuals of European descent. Nature genetics 48, 1031 (2016).

89. Howard, D.M. et al. Genome-wide meta-analysis of depression identifies 102 independent variants and highlights the importance of the prefrontal brain regions. Nature neuroscience 22, 343 (2019). spectrum disorder highlights a novel locus at 10q24. 32 and a significant overlap with schizophrenia. Molecular Autism 8, 21 (2017). 
1 91. Baselmans, B.M. et al. Multivariate genome-wide analyses of the well-being 2 spectrum. Nature genetics 51, 445-451 (2019).

3 92. Lane, J.M. et al. Biological and clinical insights from genetics of insomnia $4 \quad$ symptoms. Nature genetics 51, 387-393 (2019).

5 93. Hill, W. et al. A combined analysis of genetically correlated traits identifies 187 loci and a role for neurogenesis and myelination in intelligence. Molecular Psychiatry 24, 169-181 (2019).

8 94. Supekar, K., Cai, W., Krishnadas, R., Palaniyappan, L. \& Menon, V. Dysregulated 9 brain dynamics in a triple-network saliency model of schizophrenia and its relation to psychosis. Biological psychiatry 85, 60-69 (2019).

11 95. Zheng, H. et al. The altered triple networks interaction in depression under resting state based on graph theory. BioMed research international 2015.

13 96. Uddin, L.Q. et al. Salience network-based classification and prediction of symptom severity in children with autism. JAMA psychiatry 70, 869-879 (2013).

97. Ripke, S. et al. Biological insights from 108 schizophrenia-associated genetic loci. Nature 511, 421 (2014).

98. Kichaev, G. et al. Leveraging polygenic functional enrichment to improve GWAS power. The American Journal of Human Genetics 104, 65-75 (2019).

99. Liu, M. et al. Association studies of up to 1.2 million individuals yield new insights into the genetic etiology of tobacco and alcohol use. Nature genetics 51, 237-244 (2019).

100. van der Meer, D. et al. Brain scans from 21,297 individuals reveal the genetic architecture of hippocampal subfield volumes. Molecular Psychiatry, in press.

(2018).

25 101. Savage, J.E. et al. Genome-wide association meta-analysis in 269,867 individuals identifies new genetic and functional links to intelligence. Nature Genetics 50,

28 102. Bulik-Sullivan, B. et al. An atlas of genetic correlations across human diseases and traits. Nature Genetics 47, 1236-1241 (2015).

30 103. Zhao, B. et al. Common genetic variation influencing human white matter microstructure. bioRxiv (2020). 
1 104. Uddin, L., Nomi, J., Hébert-Seropian, B., Ghaziri, J. \& Boucher, O. Structure and Function of the Human Insula. Journal of Clinical Neurophysiology: Official Publication of the American Electroencephalographic Society 34, 300-306 (2017).

105. Binkofski, F.C., Klann, J. \& Caspers, S. On the neuroanatomy and functional role of the inferior parietal lobule and intraparietal sulcus. in Neurobiology of language 35-47 (Elsevier, 2016).

106. Cappelletti, M., Lee, H.L., Freeman, E.D. \& Price, C.J. The role of right and left parietal lobes in the conceptual processing of numbers. Journal of Cognitive Neuroscience 22, 331-346 (2010).

107. Koenigs, M., Barbey, A.K., Postle, B.R. \& Grafman, J. Superior parietal cortex is critical for the manipulation of information in working memory. Journal of Neuroscience 29, 14980-14986 (2009).

108. Artemenko, C., Sitnikova, M.A., Soltanlou, M., Dresler, T. \& Nuerk, H.-C. functional lateralization of arithmetic processing in the intraparietal sulcus is associated with handedness. Scientific reports 10, 1-11 (2020).

109. Bethmann, A., Tempelmann, C., De Bleser, R., Scheich, H. \& Brechmann, A. Determining language laterality by $\mathrm{fMRI}$ and dichotic listening. Brain research 1133, 145-157 (2007).

110. Szaflarski, J.P. et al. Language lateralization in left-handed and ambidextrous people: fMRI data. Neurology 59, 238-244 (2002).

111. Knecht, S. et al. Handedness and hemispheric language dominance in healthy humans. Brain 123, 2512-2518 (2000).

112. Urger, S.E. et al. The superior longitudinal fasciculus in typically developing children and adolescents: diffusion tensor imaging and neuropsychological correlates. Journal of Child Neurology 30, 9-20 (2015).

113. Vestergaard, M. et al. White matter microstructure in superior longitudinal fasciculus associated with spatial working memory performance in children. Journal of Cognitive Neuroscience 23, 2135-2146 (2011).

114. Klarborg, B. et al. Sustained attention is associated with right superior longitudinal fasciculus and superior parietal white matter microstructure in children. Human Brain Mapping 34, 3216-3232 (2013). 
1 115. Hamilton, L.S. et al. Reduced white matter integrity in attention-deficit hyperactivity disorder. Neuroreport 19, 1705 (2008).

3 116. Rizio, A.A. \& Diaz, M.T. Language, aging, and cognition: Frontal aslant tract and superior longitudinal fasciculus contribute to working memory performance in older adults. Neuroreport 27, 689 (2016).

6 117. Madhavan, K.M., McQueeny, T., Howe, S.R., Shear, P. \& Szaflarski, J. Superior longitudinal fasciculus and language functioning in healthy aging. Brain research 1562, 11-22 (2014).

9 118. Cavanna, A.E. \& Trimble, M.R. The precuneus: a review of its functional anatomy and behavioural correlates. Brain 129, 564-583 (2006).

11 119. Bijsterbosch, J.D., Beckmann, C.F., Woolrich, M.W., Smith, S.M. \& Harrison, S.J. The relationship between spatial configuration and functional connectivity of brain regions revisited. Elife 8, e44890 (2019).

120. Consortium, C.-D.G.o.t.P.G. Identification of risk loci with shared effects on five major psychiatric disorders: a genome-wide analysis. The Lancet 381, 1371-1379 (2013).

121. Barber, A.D. et al. Connectivity supporting attention in children with attention deficit hyperactivity disorder. Neuroimage: clinical 7, 68-81 (2015).

122. Mokobane, M., Pillay, B.J. \& Meyer, A. Fine motor deficits and attention deficit hyperactivity disorder in primary school children. South African Journal of Psychiatry 25(2019).

123. DeLong, M. et al. Role of basal ganglia in limb movements. Human neurobiology 2, 235-244 (1984).

Alexander, G.E. \& Crutcher, M.D. Preparation for movement: neural representations of intended direction in three motor areas of the monkey. Journal of neurophysiology 64, 133-150 (1990).

125. Gain, U. The cognitive function and the framework of the functional hierarchy. Applied Computing and Informatics (2018).

29 126. Wang, L., Song, M., Jiang, T., Zhang, Y. \& Yu, C. Regional homogeneity of the 30 resting-state brain activity correlates with individual intelligence. Neuroscience letters 488, 275-278 (2011). 
1 127. de Leeuw, C.A., Mooij, J.M., Heskes, T. \& Posthuma, D. MAGMA: generalized gene-set analysis of GWAS data. PLoS Computational Biology 11, e1004219 (2015).

4 128. Atkinson-Clement, C., Pinto, S., Eusebio, A. \& Coulon, O. Diffusion tensor imaging in Parkinson's disease: review and meta-analysis. Neuroimage: Clinical 16, 98-110 (2017).

129. Muralidharan, A. et al. Physiological changes in the pallidum in a progressive model of Parkinson's disease: Are oscillations enough? Experimental neurology 279, 187-196 (2016).

130. Criaud, M. et al. Contribution of insula in Parkinson's disease: A quantitative meta-analysis study. Human brain mapping 37, 1375-1392 (2016).

131. Cheng, Y.-C. et al. Genome-wide association analysis of young-onset stroke identifies a locus on chromosome 10q25 near HABP2. Stroke 47, 307-316 (2016).

132. Wang, Q. et al. A Bayesian framework that integrates multi-omics data and gene networks predicts risk genes from schizophrenia GWAS data. Nature neuroscience 22, 691 (2019).

133. Axelrud, L.K. et al. Genetic risk for Alzheimer's disease and functional brain connectivity in children and adolescents. Neurobiology of aging 82, 10-17 (2019).

134. Karch, C.M. \& Goate, A.M. Alzheimer's disease risk genes and mechanisms of disease pathogenesis. Biological psychiatry 77, 43-51 (2015).

135. Zhu, Z., Lin, Y., Li, X., Driver, J.A. \& Liang, L. Shared genetic architecture between metabolic traits and Alzheimer's disease: a large-scale genome-wide cross-trait analysis. Human genetics 138, 271-285 (2019).

136. Varma, V.R. et al. Brain and blood metabolite signatures of pathology and progression in Alzheimer disease: A targeted metabolomics study. PLoS medicine 15, e1002482 (2018).

137. Finucane, H.K. et al. Partitioning heritability by functional annotation using genome-wide association summary statistics. Nature genetics 47, 1228-1235 (2015).

138. Kundaje, A. et al. Integrative analysis of 111 reference human epigenomes. Nature 518, 317 (2015). 
1 139. Fullard, J.F. et al. An atlas of chromatin accessibility in the adult human brain.

$2 \quad$ Genome research 28, 1243-1252 (2018).

3 140. Aguet, F. et al. The GTEx Consortium atlas of genetic regulatory effects across

4 human tissues. BioRxiv, 787903 (2019).

5 141. Schmitt, A.D. et al. A compendium of chromatin contact maps reveals spatially active regions in the human genome. Cell Reports 17, 2042-2059 (2016).

7 142. Giusti-Rodriguez, P.M. \& Sullivan, P.F. Using three-dimensional regulatory chromatin interactions from adult and fetal cortex to interpret genetic results for

$9 \quad$ psychiatric disorders and cognitive traits. BioRxiv, 406330 (2019).

10143 . Jung, l. et al. A compendium of promoter-centered long-range chromatin 11 interactions in the human genome. Nature genetics, 1-8 (2019).

12 144. Song, M. et al. Mapping cis-regulatory chromatin contacts in neural cells links neuropsychiatric disorder risk variants to target genes. Nature genetics 51, 1252 (2019).

15 145. Song, M. et al. 3D Epigenomic Characterization Reveals Insights Into Gene Regulation and Lineage Specification During Corticogenesis. bioRxiv (2020).

17 146. Bycroft, C. et al. The UK Biobank resource with deep phenotyping and genomic data. Nature 562, 203-209 (2018).

147. Jiang, L. et al. A resource-efficient tool for mixed model association analysis of large-scale data. Nature genetics 51, 1749 (2019).

21 148. Purcell, S. et al. PLINK: a tool set for whole-genome association and population-based linkage analyses. The American Journal of Human Genetics 81, 559-575 (2007).

149. Willer, C.J., Li, Y. \& Abecasis, G.R. METAL: fast and efficient meta-analysis of genomewide association scans. Bioinformatics 26, 2190-2191 (2010).

26 150. Consortium, I.H. Integrating common and rare genetic variation in diverse 27 human populations. Nature 467, 52-58 (2010).

28 151. Liberzon, A. et al. Molecular signatures database (MSigDB) 3.0. Bioinformatics 27, $1739-1740$ (2011). 
1 Imaging phenotypes and datasets. The rsfMRI datasets were consistently processed

2 following the procedures in UK Biobank imaging pipeline ${ }^{9}$. Details about image

3 acquisition, preprocessing, and phenotype generation in each dataset can be found in

4 Supplementary Note. Following the previous study ${ }^{31}$, we generated two groups of

5 phenotypes, including 76 node amplitude traits reflecting the spontaneous neuronal

6 activity, and 1,695 pairwise functional connectivity traits quantifying co-activity for node

7 pairs, as well as 6 global functional connectivity measures to summarize all pairwise

8 functional connectivity. To aid interpretation of these phenotypes, the functional brain

9 regions characterized in ICA were labelled using the automated anatomical labeling

10 atlas $^{51}$ (Supplementary Table 24) and were mapped onto major functional networks

11 defined in Yeo, et al. ${ }^{14}$ and Finn, et al. ${ }^{12}$ (Supplementary Figs. 25-26). The assigned

12 location and functional networks are provided in Supplementary Table 25. Details of

13 our mapping procedures are provided in Supplementary Note. For each continuous

14 phenotype or covariate variable, values greater than five times the median absolute

15 deviation from the median value were removed. We analyzed the following nine

16 datasets separately: 1) the UKB discovery GWAS, which used data of individuals of

17 British ancestry ${ }^{146}$ in the UKB study $(n=34,691)$; 2$)$ four European validation GWAS: UKB

18 White but Non-British (UKBW, $n=1,970), \mathrm{ABCD}$ European ( $\mathrm{ABCDE}, n=3,821), \mathrm{HCP}(n=$

19 495), and PNC ( $n=510) ; 3)$ two non-European UKB validation GWAS: UKB Asian (UKBA,

$20 n=446$ ) and UKB Black (UKBBL, $n=232$ ); and 4) two non-European non-UKB validation

21 GWAS, including ABCD Hispanic ( $\mathrm{ABCDH}, n=768)$ and ABCD African American (ABCDA, $n$

22 = 1,257). See Supplementary Table 26 for a summary of these datasets and

23 demographic information. The assignment of ancestry in UKB was based on

24 self-reported ethnicity (Data-Field 21000), which was verified in Bycroft, et al. ${ }^{146}$. The

25 ancestry in $A B C D$ was assigned by combining the self-reported ethnicity and ancestry

26 inference results as in Zhao, et al. ${ }^{103}$.

28 GWAS discovery and validation. Details of genotyping and quality controls can be found 29 in Supplementary Note. SNP heritability was estimated by GCTA ${ }^{52}$ using all autosomal 30 SNPs in the UKB British cohort. We adjusted the effects of age (at imaging),

31 age-squared, sex, age-sex interaction, age-squared-sex interaction, imaging site, and the

32 top 40 genetic principle components (PCs). Genome-wide association analysis was 
1 performed in linear mixed effect model using fastGWA ${ }^{147}$, while adjusting the same set

2 of covariates as in GCTA. GWAS were also separately performed via Plink ${ }^{148}$ in the eight

3 validation datasets, including UKBW, UKBBL, UKBA, ABCDA, ABCDH, ABCDE, $H C P$, and

4 PNC, where the effects of age, age-squared, sex, imaging sites (if applicable), scanners (if

5 applicable), age-sex interaction, age-squared-sex interaction, and top ten genetic PCs

6 were adjusted.

8 To validate results in the UKB British discovery GWAS, meta-analysis was performed 9 using the sample-size weighted approach via METAL ${ }^{149}$. We examined whether the 10 locus-level associations detected in the British GWAS can be replicated in the 1) 11 meta-analyzed four European validation GWAS (UKBW, ABCDE, HCP, and PNC); 2) 12 meta-analyzed four non-European validation GWAS (UKBBL, UKBA, ABCDA, and ABCDH);

13 and 3) the combination of the above eight validation GWAS. Specifically, for each 14 meta-analyzed GWAS, we checked and reported the smallest $P$-value among the 15 variants within each associated locus identified in the UKB British discovery GWAS. 16 Polygenic risk scores (PRS) were constructed on eight validation datasets using Plink.

17 The BLUP effect sizes estimated from GCTA-GREML analysis in UKB British discovery 18 GWAS were used as weights in PRS construction, which accounted for the LD structures. 19 Ambiguous variants (i.e. variants with complementary alleles) were removed from 20 analysis. We tried $17 P$-value thresholds for variant selection according to their marginal $21 P$-values from fastGWA: $1,0.8,0.5,0.4,0.3,0.2,0.1,0.08,0.05,0.02,0.01,1 \times 10^{-3}, 1 \times$ $2210^{-4}, 1 \times 10^{-5}, 1 \times 10^{-6}, 1 \times 10^{-7}$, and $1 \times 10^{-8}$. The best prediction accuracy achieved by a 23 single threshold was reported for each phenotype, which was measured by the 24 additional phenotypic variation that can be explained by the polygenic profile (i.e., the 25 incremental R-squared), while adjusting for the effects of age, sex, and top ten genetic 26 PCs.

28 The shared loci and genetic correlation. The genomic loci associated with intrinsic brain 29 activity traits were defined using FUMA (version 1.3.5e). We input UKB British discovery 30 summary statistics after reweighting the $P$-values using functional information via 31 FINDOR ${ }^{98}$. To define the LD boundaries, FUMA identified independent significant 32 variants, which were defined as variants with a $P$-value smaller than the predefined 
1 threshold and were independent of other significant variants (LD $r^{2}<0.6$ ). FUMA then

2 constructed LD blocks for these independent significant variants by tagging all variants

3 in $\operatorname{LD}\left(r^{2} \geq 0.6\right)$ with at least one independent significant variant and had a MAF $\geq$

$4 \quad 0.0005$. These variants included those from the 1000 Genomes reference panel that may

5 not have been included in the GWAS. Moreover, within these significant variants,

6 independent lead variants were identified as those that were independent from each

7 other (LD $\left.r^{2}<0.1\right)$. If LD blocks of independent significant variants were close $(<250 \mathrm{~kb}$

8 based on the closest boundary variants of LD blocks), they were merged into a single

9 genomic locus. Thus, each genomic locus could contain multiple significant variants and

10 lead variants. Independent significant variants and all the variants in LD with them $\left(r^{2} \geq\right.$

11 0.6) were searched by FUMA on the NHGRI-EBI GWAS catalog (version 2019-09-24) to

12 look for previously reported associations $\left(P<9 \times 10^{-6}\right)$ with any traits. LDSC $^{102}$ software

13 (version 1.0.1) was used to estimate and test the pairwise genetic correlation. We used

14 the pre-calculated LD scores provided by LDSC, which were computed using 1000

15 Genomes European data. We used HapMap3 ${ }^{150}$ variants and removed all variants in the

16 major histocompatibility complex $(\mathrm{MHC})$ region. The summary statistics of intrinsic brain

17 activity traits were from the UKB British discovery GWAS and the resources of other

18 summary statistics were provided in Supplementary Table 9.

20 Gene-level analysis and biological annotation. Gene-based association analysis was

21 performed in UKB British participants for 18,796 protein-coding genes using MAGMA ${ }^{127}$

22 (version 1.07). Default MAGMA settings were used with zero window size around each

23 gene. We then carried out FUMA functional annotation and mapping analysis, in which

24 variants were annotated with their biological functionality and then were linked to

2535,808 candidate genes by a combination of positional, eQTL, and 3D chromatin

26 interaction mappings. Brain-related tissues/cells were selected in all options and default

27 values were used for all other parameters in FUMA. For the detected genes in MAGMA

28 and FUMA, we performed lookups in the NHGRI-EBI GWAS catalog (version 2020-02-08)

29 to explore their previously reported gene-trait associations. We performed heritability

30 enrichment analysis via partitioned LDSC $^{137}$. Baseline models were adjusted when

31 estimating and testing the enrichment scores for our tissue type and cell type specific

32 annotations. Methods to analysis chromatin data of glial and neuronal cell subtypes can 
1 be found in Zhao, et al. ${ }^{103}$. We also performed gene property analysis for the 13 GTEx ${ }^{140}$

2 v8 brain tissues via MAGMA. Specifically, we examined whether the tissue-specific gene

3 expression levels can be linked to the strength of the gene-trait association. MAGMA

4 was also used to explore the enriched biological pathways, in which we tested 500

5 curated gene sets and 9,996 Gene Ontology (GO) terms from the Molecular Signatures

6 Database ${ }^{151}$ (MSigDB, version 7.0). Additional gene mapping was performed using 14

7 Hi-C datasets of brain tissue and cell types from five recent studies, including 1) the

8 promoter capture $\mathrm{Hi}-\mathrm{C}(\mathrm{PCHi}-\mathrm{C})$ data of hippocampus and dorsolateral prefrontal cortex

9 (DLPFC) $)^{143}$; 2) the Hi-C data of hippocampus and DLPFC ${ }^{141}$; 3) the Hi-C data from fetal

10 and adult cortices ${ }^{142}$, restricting to the high confidence interactions; 4 ) the PCHi-C data

11 of primary astrocytes and three types of induced pluripotent stem cell (iPSC)-derived

12 neurons $^{144}$ (cortical, hippocampal, and motor); and 5) proximity ligation assisted

13 chromatin immunoprecipitation (PLAC-seq) data on sorted fetal neuron cells ${ }^{145}$,

14 including radial glial cells, intermediate progenitor cells, neurons, and interneurons. For

15 interaction intensity cutoffs, we used 2 for the $-\log 10(P)$ used in datasets of Jung, et al.

$16143,0.05$ for the $q$-value in Schmitt, et al. ${ }^{141}$ and Giusti-Rodriguez and Sullivan ${ }^{142}, 5$ for

17 the Chicago score in Song, et al. ${ }^{144}$, and 0.01 for the FDR in Song, et al. ${ }^{145}$.

19 Code availability

20 We made use of publicly available software and tools listed in URLs. Other codes used in

21 our analyses are available upon reasonable request.

\section{Data availability}

24 Our GWAS summary statistics can be downloaded at https://github.com/BIG-S2/GWAS.

25 The individual-level data used in the present study can be obtained from four publicly

26 accessible data resources: UK Biobank (http://www.ukbiobank.ac.uk/resources/), ABCD

27 (https://abcdstudy.org/), HCP (https://www.humanconnectome.org/), and PNC

28 (https://www.med.upenn.edu/bbl/philadelphianeurodevelopmentalcohort.html). Our

29 results can also be easily browsed through our knowledge portal https://bigkp.org/. 


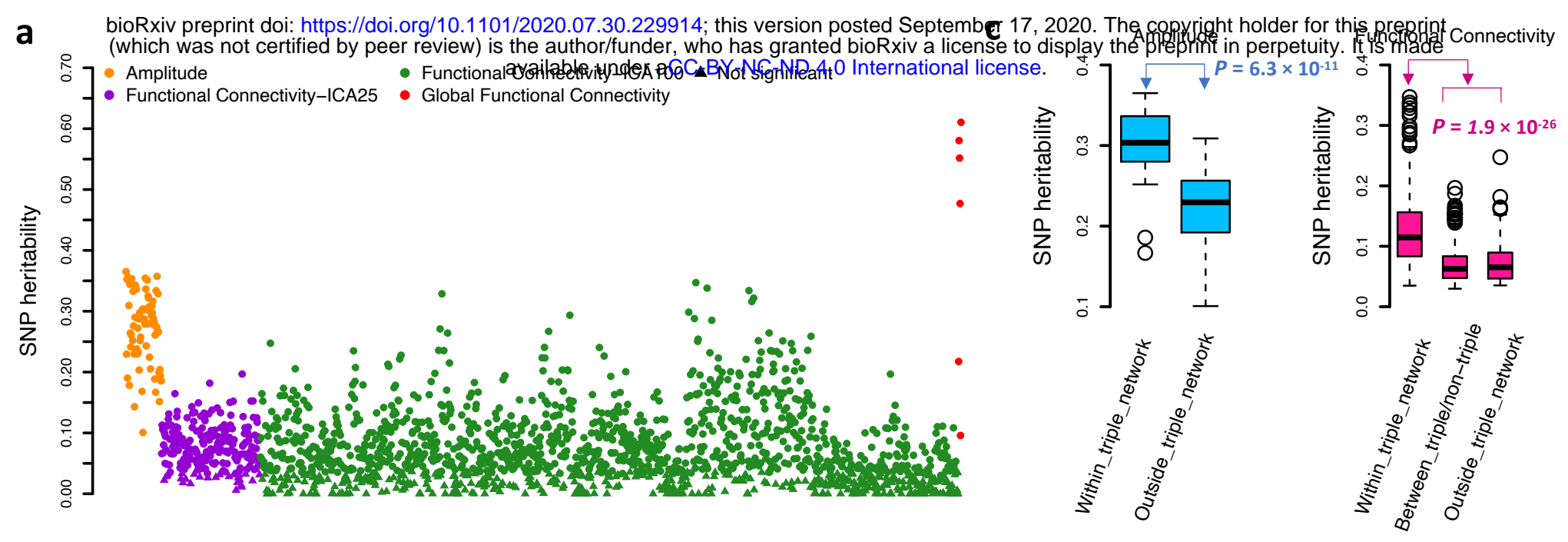

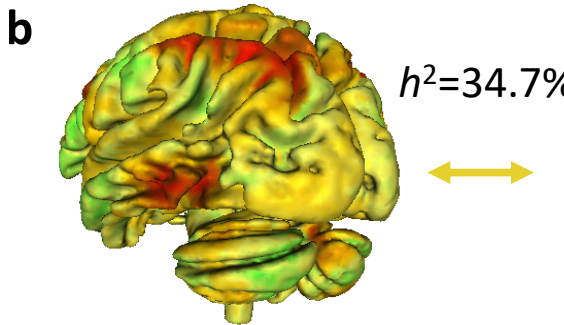

(Inferior parietal, Angular) (Central executive, Attention) (Default mode, Central executive)

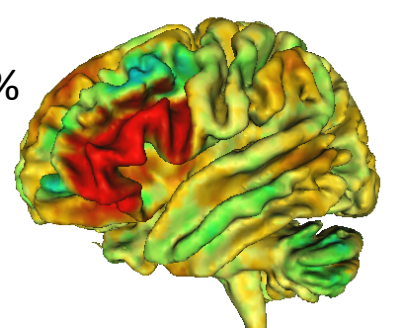

(Inferior frontal)

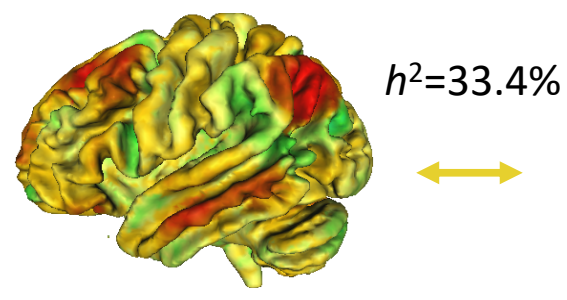

(Precuneus, Angular, Middle cingulate)
(Superior frontal, Middle frontal)

(Central executive,

(Default mode, Central executive) Salience, Default mode)

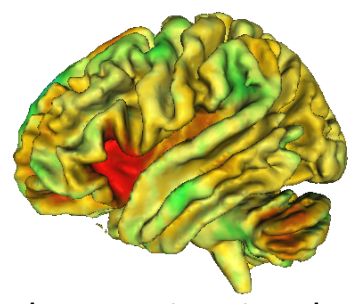

(Insula, Anterior cingulate)

(Salience,

Default mode) $h^{2}=32.2 \%$

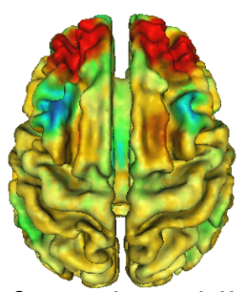

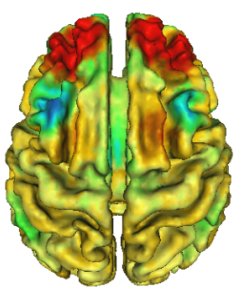

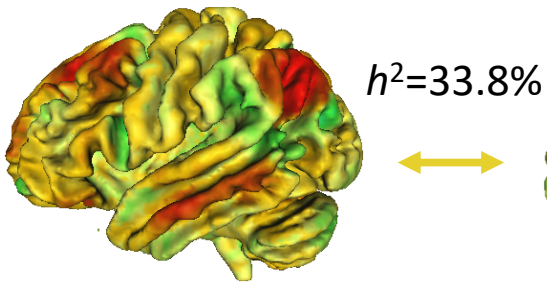

(Precuneus, Angular, Middle cingulate)

(Default mode, Central executive)

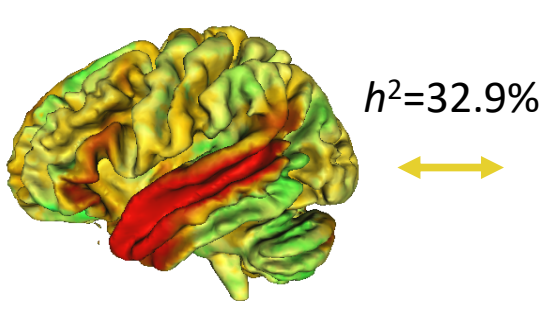

(Middle temporal, Temporal pole) (Default mode)

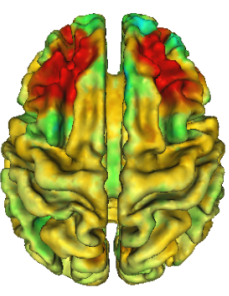

(Superior frontal, Middle frontal) (Salience, Default mode)

.




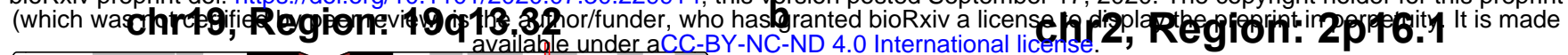

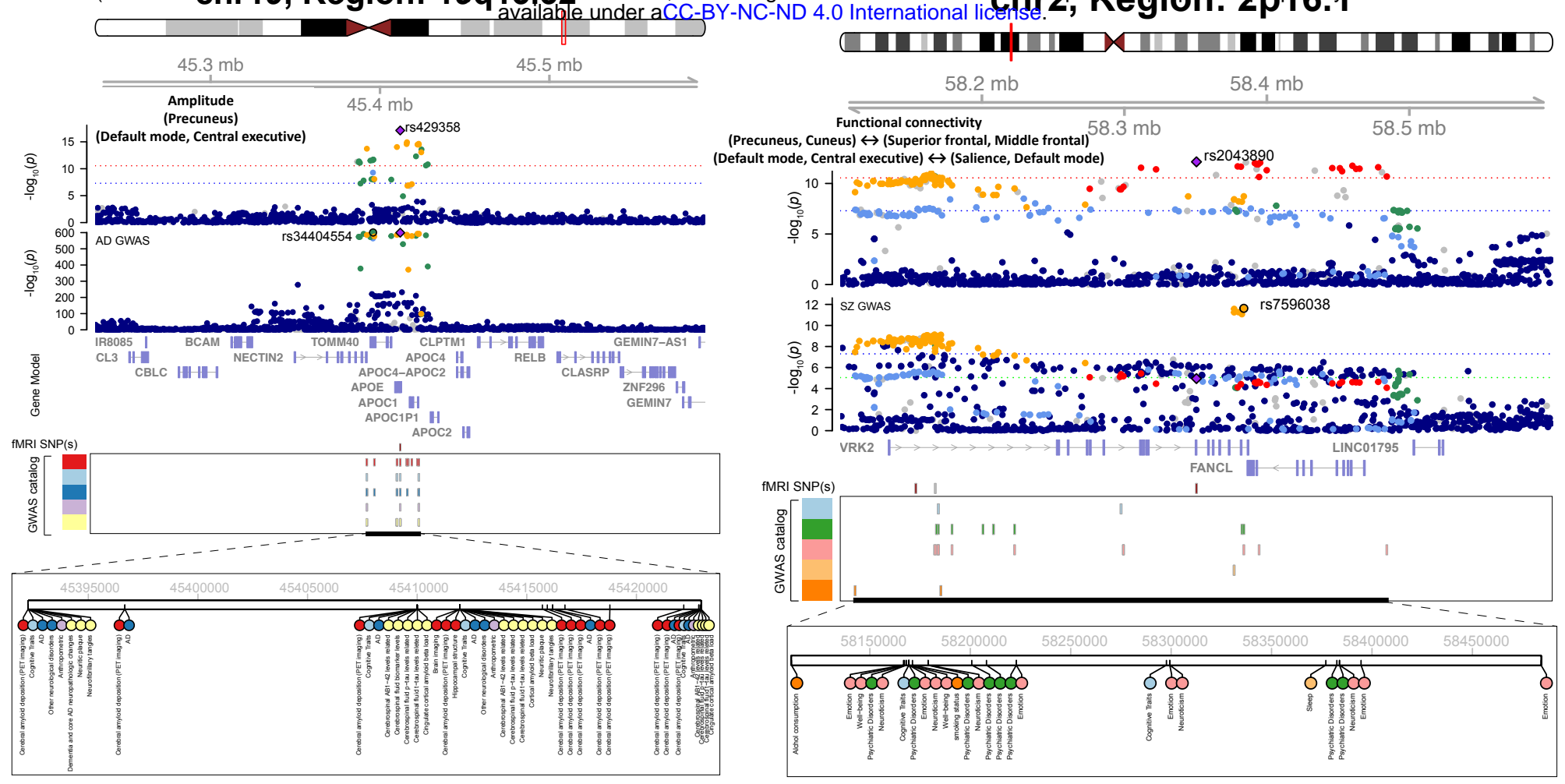

C chr2, Region: 2q14.1
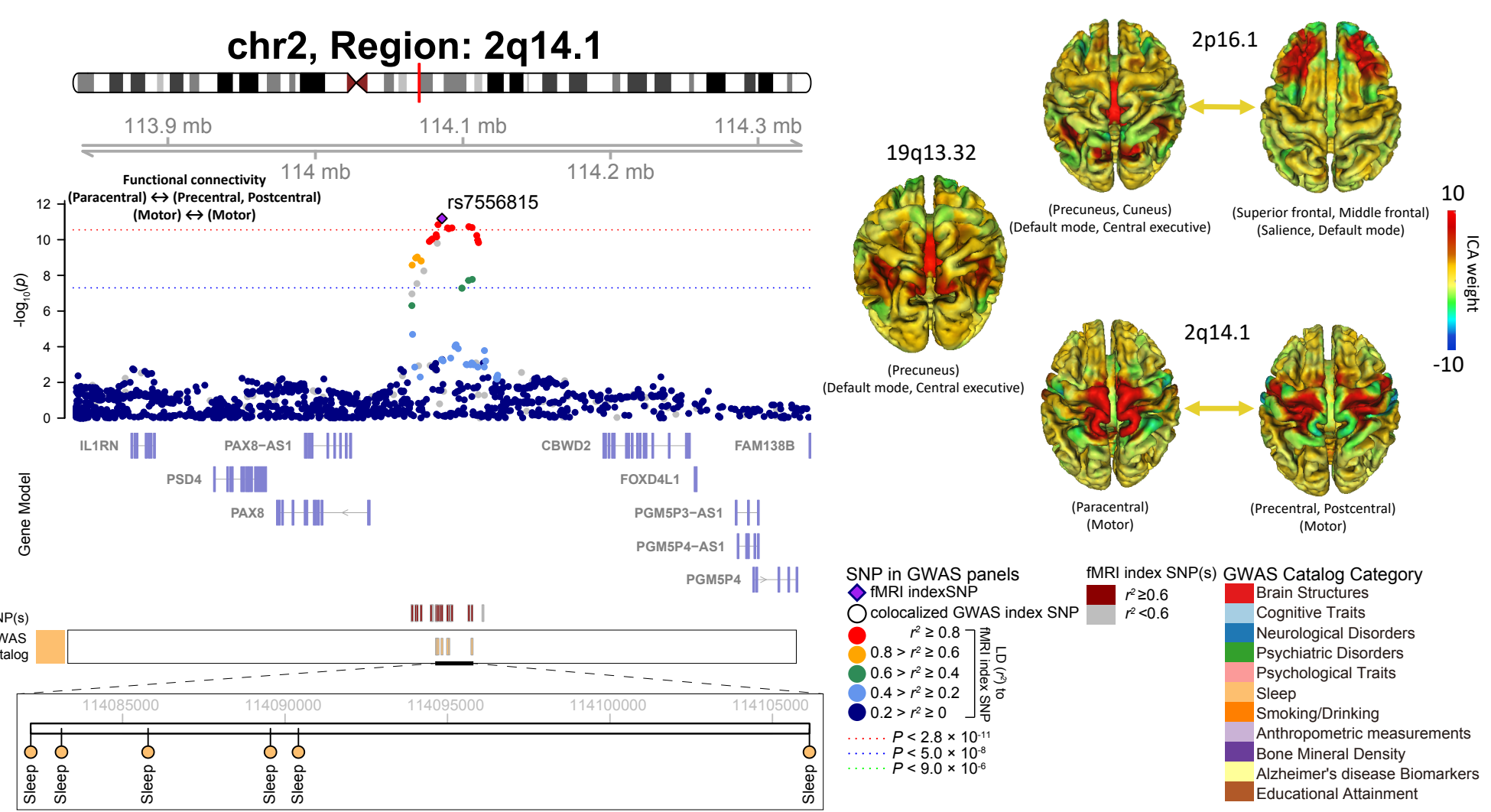

Figure 2: Selected genetic loci that associated with both rsfMRI traits of brain activity and other brain-related complex traits and disorders. We highlight local colocalization (LD $\left.r^{2} \geq 0.6\right)$ in a) $19 \mathrm{q} 13.32$ (colocalized with Alzheimer's disease); b) 2p16.1 (with schizophrenia); and c) 2q14.1 (with sleep). For example, in 19q13.32, we observed colocalization between the amplitude of the precuneus region in the default mode and central executive networks with Alzheimer's disease. Location and functional network of the displayed three rsfMRI traits are illustrated on the bottom right. More examples of the shared genetic loci and the involved rsfMRI traits can be found in Supplementary Figures 4-12. 
bioRxiv preprint doi: https://doi.org/10.1101/2020.07.30.229914; this version posted September 17, 2020. The copyright holder for this preprint (which was not certified by peer review) is the author/funder, who has granted bioRxiv a license to display the preprint in perpetuity. It is made available under aCC-BY-NC-ND 4.0 International license.

\section{a}

(Middle frontal; Cerebellum) $\leftrightarrow$ (SupraMarginal; Inferior parietal) (Middel frontal) $\leftrightarrow$ (Middle temporal)

(Precuneus; Angular; Middle cingulate) $\leftrightarrow$ (Precuneus)

(Precuneus; Angular; Middle cingulate) $\leftrightarrow$ (Superior frontal; Middle frontal) (Angular; Middle temporal) $\leftrightarrow$ (Superior frontal; Middle frontal) (Inferior frontal; Precentral) $\leftrightarrow$ (Superior frontal; Middle frontal)

(Precuneus; Angular; Middle cingulate) $\leftrightarrow$ (Middle temporal)

(Angular; Middle temporal) $\leftrightarrow$ (Middle temporal; Angular)

(Precuneus; Angular; Middle cingulate) $\leftrightarrow$ (Middle temporal; Angular)

(Precuneus) $\leftrightarrow$ (Inferior temporal; Middle temporal)

(Inferior temporal; Middle temporal) $\leftrightarrow$ (Middle temporal)

(Calcarine; Lingual; Cuneus) $\leftrightarrow$ (Cuneus; Superior occipital) (Precuneus; Cuneus) $\leftrightarrow$ (Precuneus)

(Calcarine; Lingual; Cuneus) $\leftrightarrow$ (Calcarine; Lingual) (Middle frontal) $\leftrightarrow$ (Middle temporal; Angular) (Middle frontal) $\leftrightarrow$ (Middle temporal)

(Middle temporal; Temporal pole) $\leftrightarrow$ (SupraMarginal; Inferior parietal) (Precuneus; Angular; Middle cingulate) $\leftrightarrow$ (SupraMarginal; Inferior parietal)

(SupraMarginal; Inferior parietal) $\leftrightarrow$ (Middle frontal)

(SupraMarginal; Inferior parietal) $\leftrightarrow$ (Inferior temporal; Inferior occipital) (Precuneus) $\leftrightarrow$ (Middle temporal; Angular)

(Inferior parietal; Postcentral) $\leftrightarrow$ (Precuneus; Superior parietal)

\section{Brain functional connectivity}
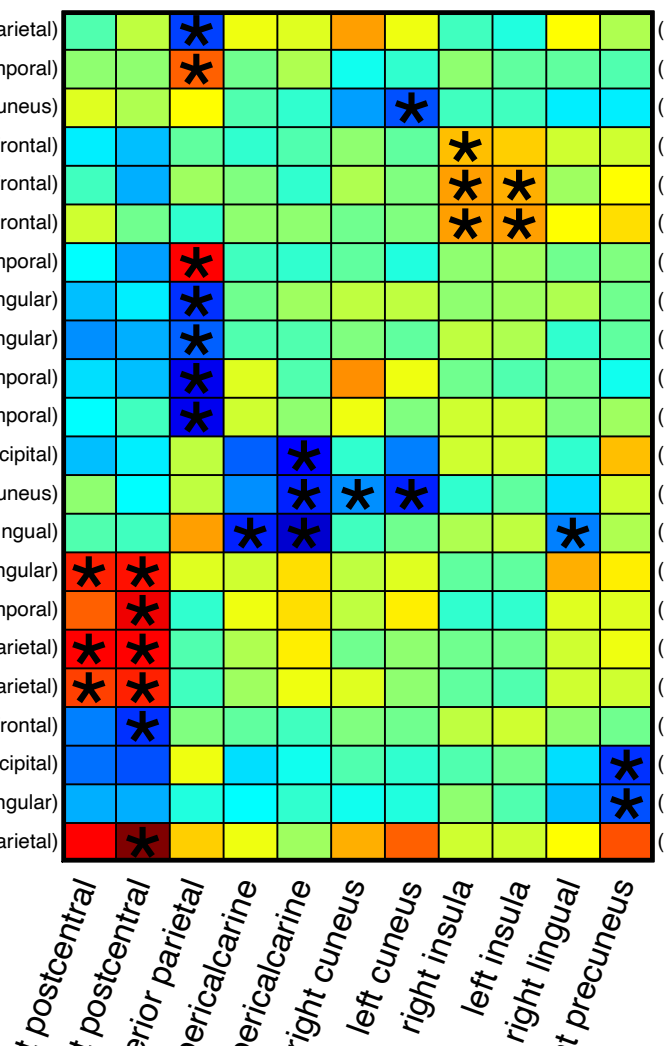

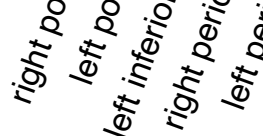

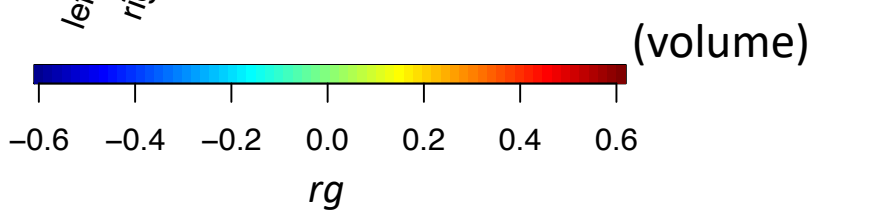
(Visual) $\leftrightarrow$ (Visual)
(Central executive; Default mode) $\leftrightarrow$ (Central executive; Salience) (Central executive) $\leftrightarrow$ (Default mode; Central executive)

(Default mode; Central executive) $\leftrightarrow$ (Default mode; Central executive) (Default mode; Central executive) $\leftrightarrow$ (Central executive; Salience; Default mode) (Default mode; Central executive) $\leftrightarrow$ (Central executive; Salience; Default mode) (Central executive) $\leftrightarrow$ (Central executive; Salience; Default mode) (Default mode; Central executive) $\leftrightarrow$ (Default mode; Central executive) (Default mode; Central executive) $\leftrightarrow$ (Default mode) (Default mode; Central executive) $\leftrightarrow$ (Default mode) (Default mode; Central executive) $\leftrightarrow$ (Attention; Visual) (Attention; Visual) $\leftrightarrow$ (Default mode; Central executive) Visual $) \leftrightarrow($ Visual $)$ (Default mode; Central executive) $\leftrightarrow$ (Default mode; Central executive)

(Central executive; Salience) $\leftrightarrow$ (Default mode) Central executive; Salience) $\leftrightarrow$ (Default mode; Central executive) (Default mode) $\leftrightarrow$ (Central executive; Salience) (Default mode; Central executive) $\leftrightarrow$ (Central executive; Salience) (Central executive; Salience) $\leftrightarrow$ (Central executive; Salience) (Central executive; Salience) $\leftrightarrow$ (Attention; Visual) (Default mode; Central executive) $\leftrightarrow$ (Default mode) (Attention; Central executive; Salience) $\leftrightarrow$ (Attention; Central executive)

\section{Functional networks}

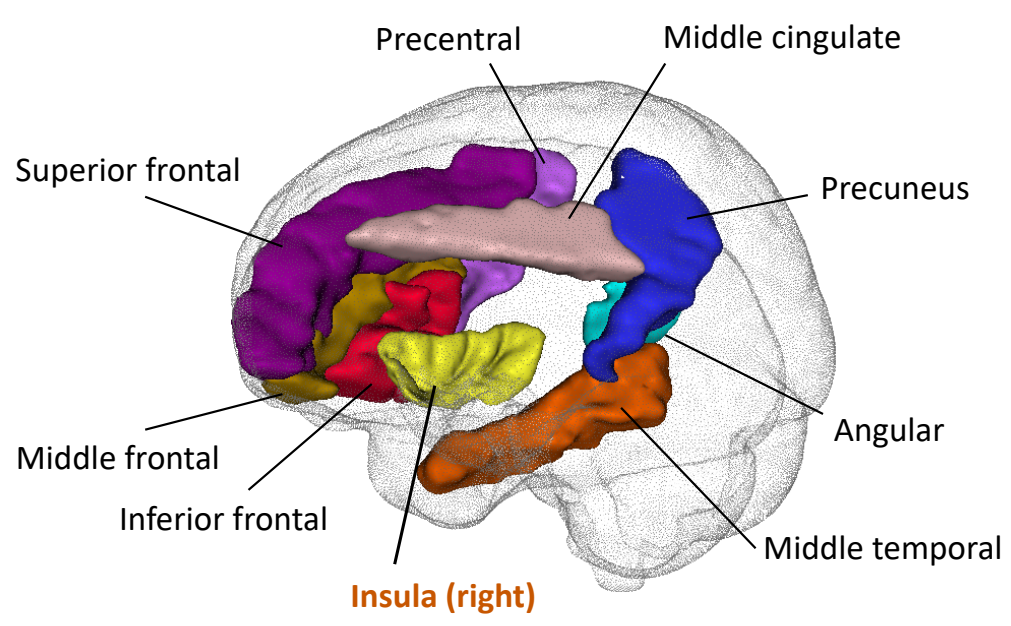

b

Figure 3: Selected pairwise genetic correlations between functional connectivity traits and regional brain volumes. a) The asterisks highlight significant associations after controlling the false discovery rate at 0.05 level. The left y-axis lists the location of functional connectivity traits, the right y-axis shows the associated functional networks, and the $\mathrm{x}$-axis provides the name of regional brain volumes. The colors represent genetic correlations (rg). b) Location of the right insula and its neighboring brain regions whose functional connectivity strengths were genetically correlated with the right insula volume. The colors describe different brain regions. c) Location of the left pericalcarine and its neighboring brain regions whose functional connectivity strengths were genetically correlated with the left pericalcarine volume. 


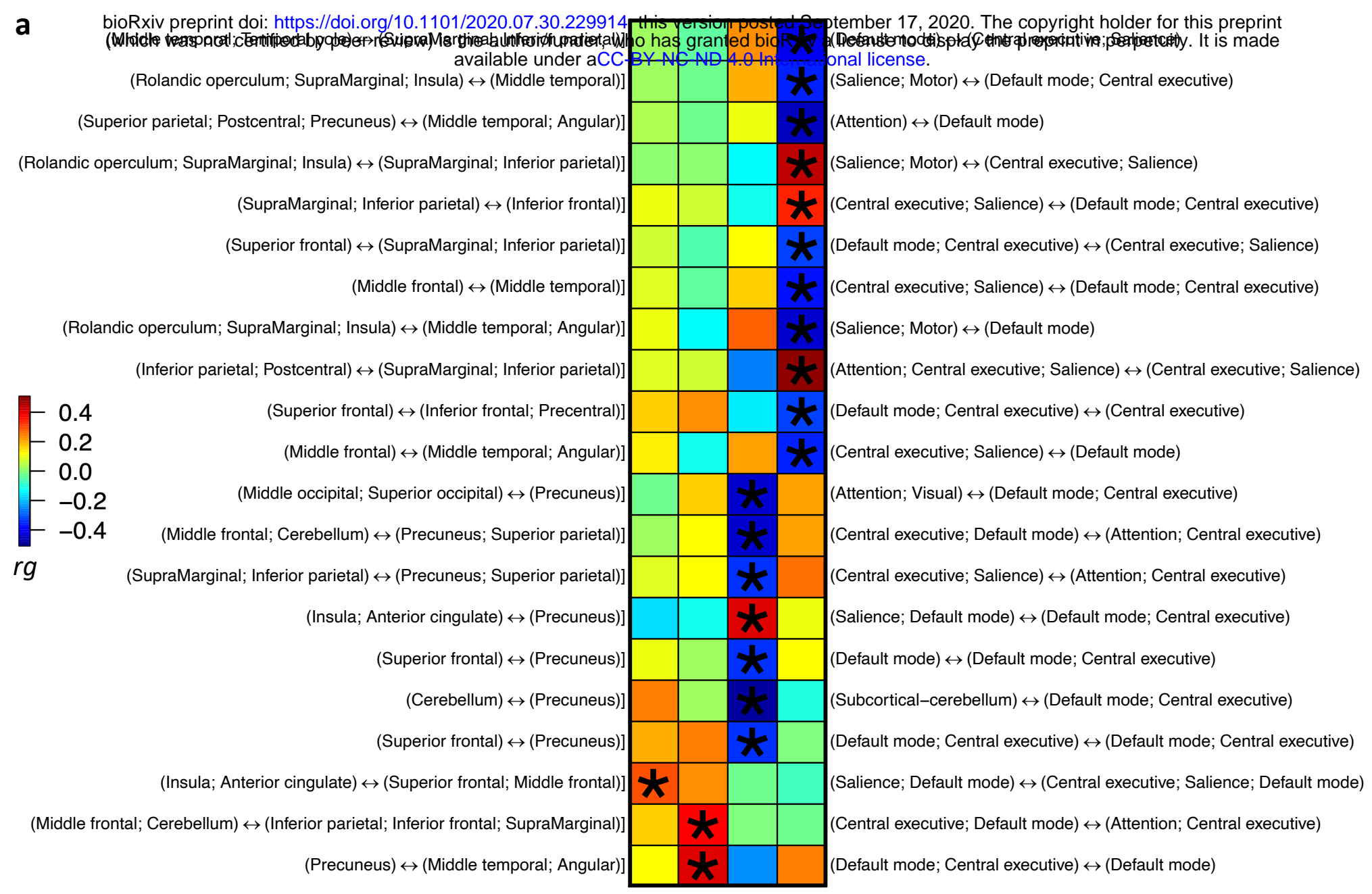

Brain functional connectivity

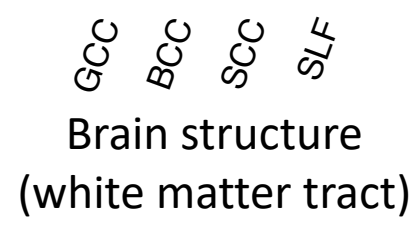

Functional networks

b

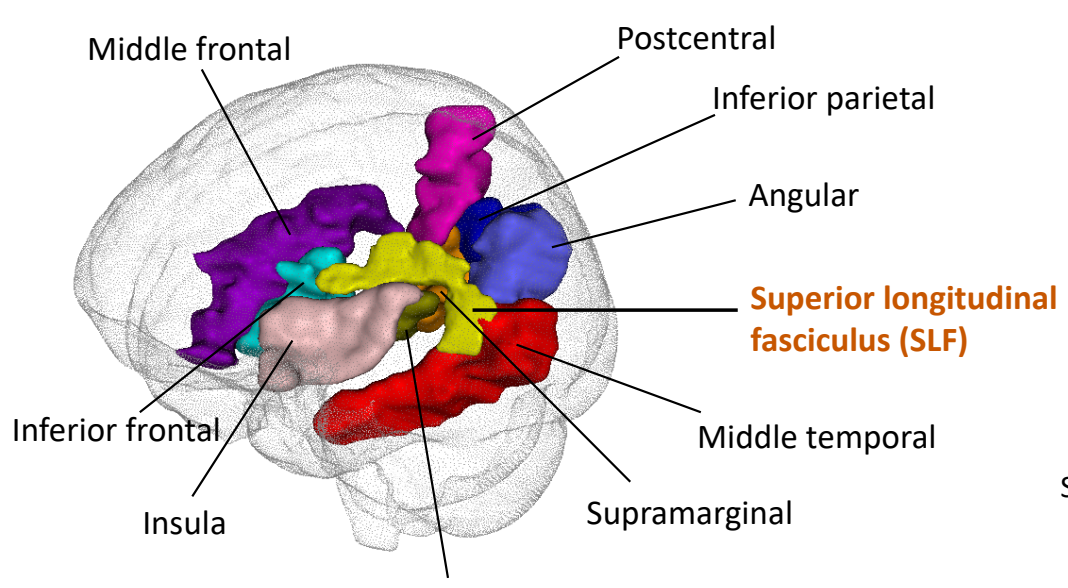

Rolandic operculum c

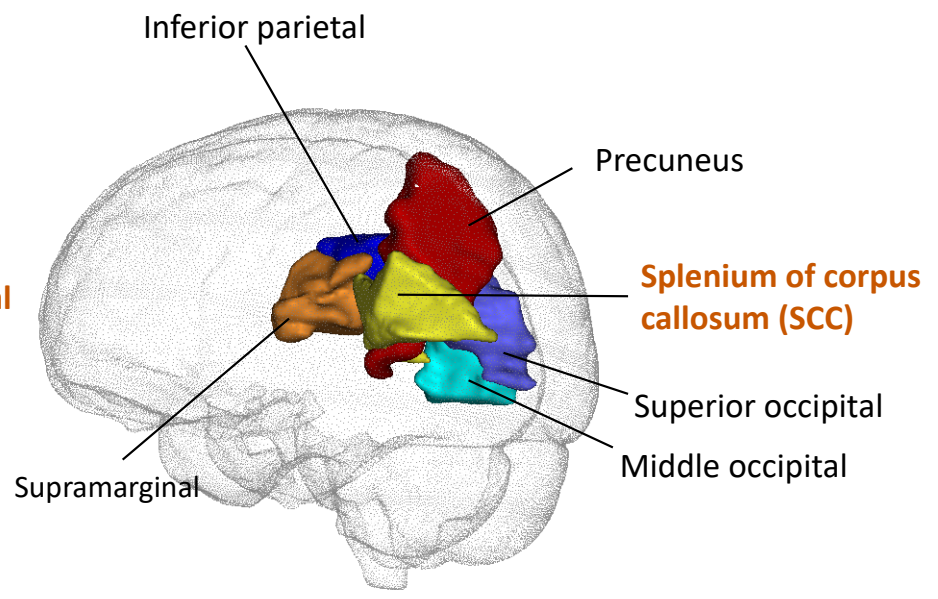

Figure 4: Selected pairwise genetic correlations between functional connectivity traits and fractional anisotropy (FA) of white matter tracts. a) The asterisks highlight significant associations after controlling the false discovery rate at 0.05 level. The left y-axis lists the location of functional connectivity traits, the right y-axis shows the associated functional networks, and the $\mathrm{x}$-axis provides the name of white matter tracts. The colors represent genetic correlations ( $\mathrm{rg}$ ). b) Location of the SLF (left part) and its neighboring brain regions whose functional connectivity strengths were genetically correlated with the FA of SLF. The colors describe different brain regions. c) Location of the SCC (left part) and its neighboring brain regions whose functional connectivity strengths were genetically correlated with the FA of SCC. 
bioRxiv preprint doi: https://doi org/10.11.01/2020.0730.22991/therember 17, 2020. The copyright holder for this preprint

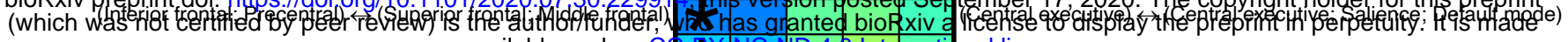

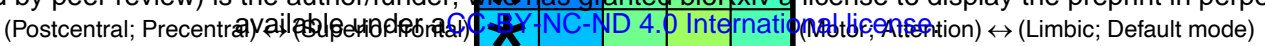

(Cerebellum) $\leftrightarrow$ (Precuneus)

(Superior parietal; Postcentral; Precuneus) $\leftrightarrow$ (Precentral; Postcentral)

(Precuneus; Angular; Middle cingulate) $\leftrightarrow$ (Cerebellum) (Postcentral; Precentral) $\leftrightarrow$ (Inferior temporal; Inferior occipital) (Lingual; Calcarine; Superior occipital) $\leftrightarrow$ (Inferior parietal; Angular; Middle temporal) (Middle occipital; Superior occipital) $\leftrightarrow$ (Precentral; Postcentral) (Superior frontal; Middle frontal) $\leftrightarrow$ (Middle temporal; Angular) (Angular; Middle temporal) $\leftrightarrow$ (Middle temporal)

0.4

0.2

0.0

$-0.2$

$-0.4$

rg

(Superior temporal) $\leftrightarrow$ (Precentral; Postcentral)

(Calcarine; Lingual; Cuneus) $\leftrightarrow$ (Middle temporal; Temporal pole) (Insula; Anterior cingulate) $\leftrightarrow$ (Inferior temporal; Inferior occipital) (Superior frontal; Middle frontal) $\leftrightarrow$ (Precuneus) (Inferior occipital; Middle occipital) $\leftrightarrow$ (Cerebellum)

(Precuneus; Angular; Middle cingulate) $\leftrightarrow$ (Precentral; Postcentral) (Postcentral; Precentral) $\leftrightarrow$ (Insula; Anterior cingulate) (Inferior parietal; Angular) $\leftrightarrow$ (Superior frontal; Middle frontal) (Superior temporal) $\leftrightarrow$ (Precentral; Superior frontal; Supp motor area) (Postcentral; Precentral) $\leftrightarrow$ (Orbital) (Cerebellum) $\leftrightarrow$ (Superior frontal; Middle frontal) (Middle frontal) $\leftrightarrow$ (Superior frontal; Middle frontal) (Precentral; Superior frontal; Supp motor area) $\leftrightarrow$ (Putamen; Caudate)

b Supplemental motor area

\section{Brain functional connectivity}

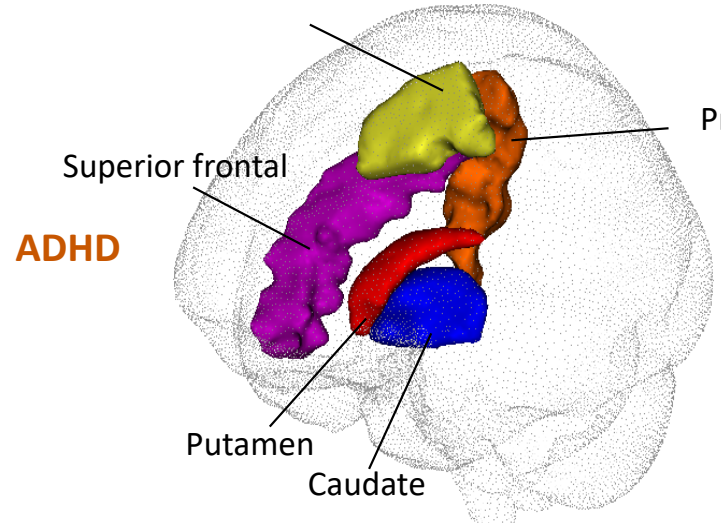

C

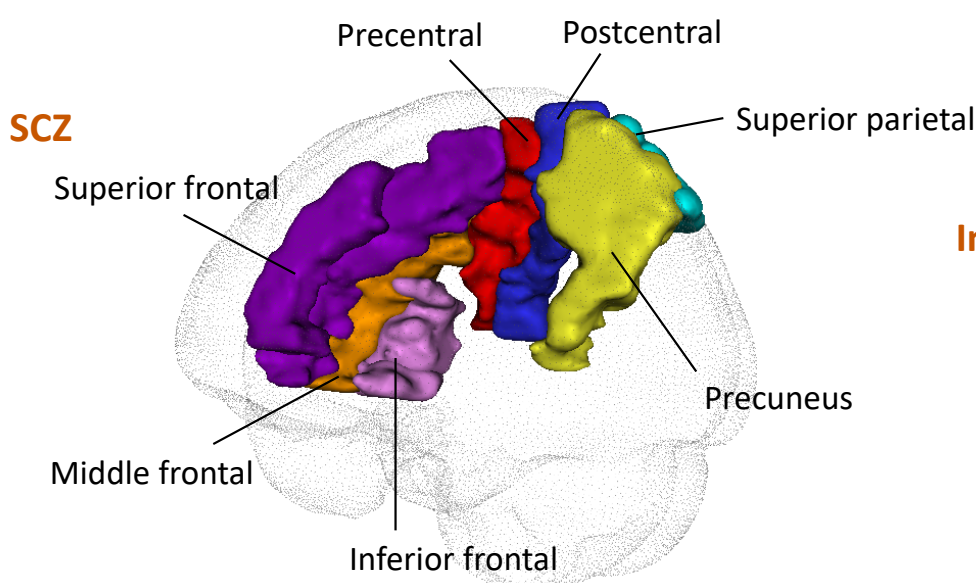

e

(Subcortical-cerebellum) $\leftrightarrow$ (Default mode; Central executive)

(Attention) $\leftrightarrow$ (Motor)

(Default mode; Central executive) $\leftrightarrow$ (Subcortical-cerebellum)

(Motor; Attention) $\leftrightarrow$ (Attention; Visual)

(Visual) $\leftrightarrow$ (Default mode; Central executive)

(Attention; Visual) $\leftrightarrow$ (Motor)

(Central executive; Salience; Default mode) $\leftrightarrow$ (Default mode)

(Default mode; Central executive) $\leftrightarrow$ (Default mode; Central executive)

(Default mode; Motor) $\leftrightarrow$ (Motor)

(Visual) $\leftrightarrow$ (Default mode)

(Salience; Default mode) $\leftrightarrow$ (Attention; Visual)

(Salience; Central executive) $\leftrightarrow$ (Default mode; Central executive)

(Visual) $\leftrightarrow$ (Subcortical-cerebellum)

(Default mode; Central executive) $\leftrightarrow$ (Motor)

(Motor) $\leftrightarrow$ (Salience; Default mode)

(Central executive; Attention) $\leftrightarrow$ (Central executive; Salience; Default mode)

(Default mode; Motor) $\leftrightarrow$ (Attention; Salience; Motor)

(Motor) $\leftrightarrow$ (Limbic)

(Subcortical-cerebellum) $\leftrightarrow$ (Salience; Default mode)

(Central executive; Salience) $\leftrightarrow$ (Central executive)

(Attention; Salience; Motor) $\leftrightarrow$ (Subcortical-cerebellum)

\section{Functional networks}

\section{d}

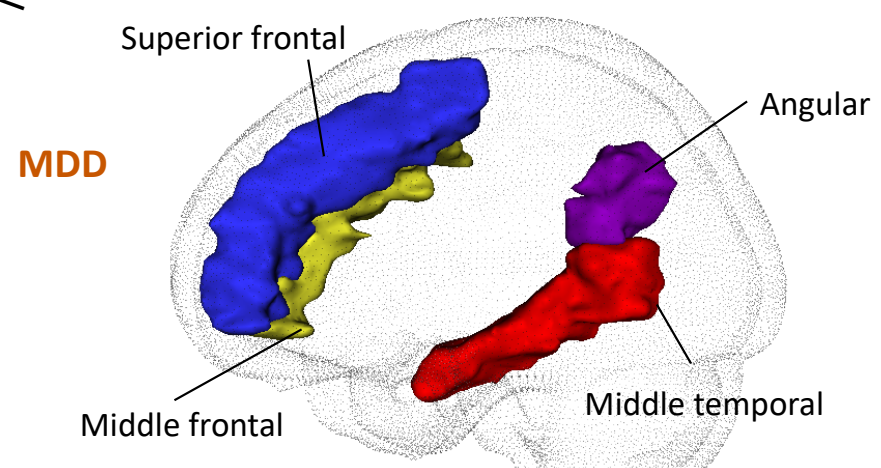

Middle cingulate Precentral

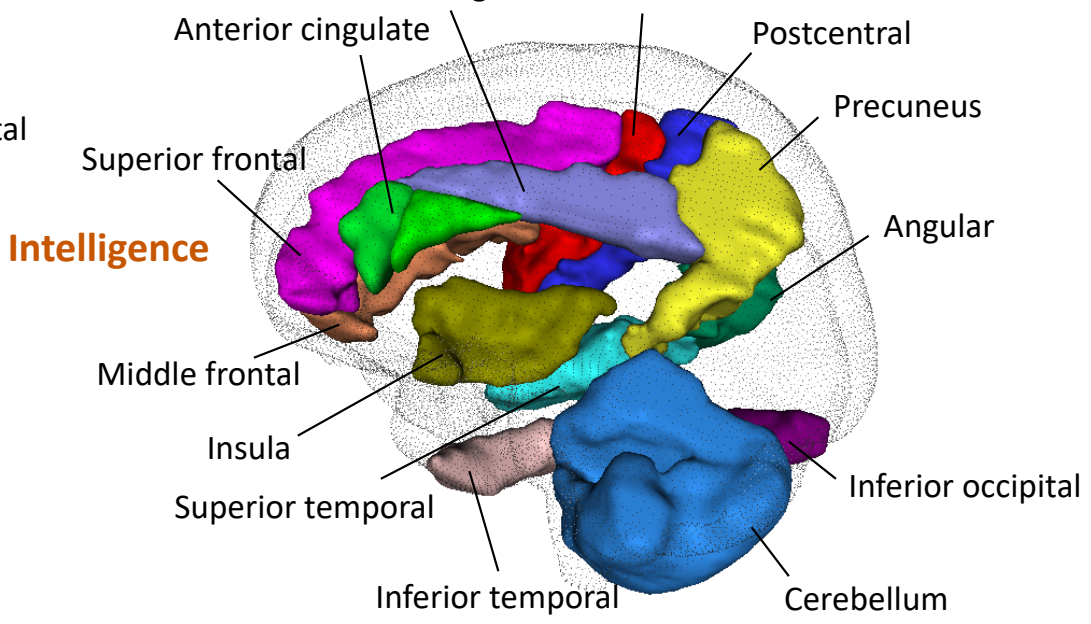

Cerebellum

Figure 5: Selected pairwise genetic correlations between functional connectivity traits and other brain-related traits/disorders. a) The asterisks highlight significant associations after controlling the false discovery rate at 0.05 level. The left y-axis lists the location of functional connectivity traits, the right y-axis shows the associated functional networks, and the x-axis provides the name of other brain-related traits/disorders. The colors represent genetic correlations (rg). b-e) Location of the brain regions whose functional connectivity strengths were genetically correlated with b) attention-deficit/hyperactivity disorder (ADHD); c) schizophrenia $(\mathrm{SCZ})$; d) major depressive disorder (MDD); and e) intelligence. The colors describe different brain regions. 